\title{
Probabilistic Description Logics under the Distribution Semantics
}

\author{
Fabrizio Riguzzi $^{\mathrm{a}}$, Elena Bellodi ${ }^{\mathrm{b}}$, Evelina Lamma $^{\mathrm{b}}$, Riccardo Zese $^{\mathrm{b}}$ \\ ${ }^{a}$ Dipartimento di Matematica e Informatica - University of Ferrara, Via Saragat 1, I-44122, Ferrara, Italy \\ ${ }^{b}$ Dipartimento di Ingegneria - University of Ferrara, Via Saragat 1, I-44122, Ferrara, Italy
}

\begin{abstract}
Representing uncertain information is crucial for modeling real world domains. In this paper we present a technique for the integration of probabilistic information in Description Logics (DLs) that is based on the distribution semantics for probabilistic logic programs. In the resulting approach, that we called DISPONTE, the axioms of a probabilistic knowledge base $(\mathrm{KB})$ can be annotated with a real number between 0 and 1 . A probabilistic knowledge base then defines a probability distribution over regular KBs called worlds and the probability of a given query can be obtained from the joint distribution of the worlds and the query by marginalization. We present the algorithm BUNDLE for computing the probability of queries from DISPONTE knowledge bases. The algorithm exploits an underlying DL reasoner, such as Pellet, that is able to return explanations for queries. The explanations are encoded in a Binary Decision Diagram from which the probability of the query is computed. The experimentation of BUNDLE on probabilistic knowledge bases shows that it can handle knowledge bases of realistic size.
\end{abstract}

\section{Introduction}

Representing uncertain information is of foremost importance in order to effectively model real world domains. This has been fully recognized in the field of Artificial Intelligence where uncertainty has been the focus of much research since its beginnings. In particular, the integration of logic and probability allows to model complex domains with many entities interconnected by uncertain relationships. This problem has been investigated by various authors both in the general case of first order logic (Nilsson, 1986; Halpern, 1990; Bacchus, 1990) and in the case of restricted logics, such as Description Logics (DLs) and Logic Programming (LP).

DLs are fragments of first order logic particularly useful for knowledge representation. They are at the basis of the Semantic Web. The Web Ontology Language (OWL) is a family of languages that are syntactic variants of various DLs. Many proposals have appeared on the combination of probability theory and DLs (Heinsohn, 1994; Jaeger, 1994; Koller et al., 1997; Lukasiewicz, 2008; Luna et al., 2011). Probabilistic DLs play an important role in the Semantic Web where knowledge may come from different sources and may have different reliability.

In LP, the distribution semantics (Sato, 1995) has emerged as one of the most effective approaches for representing probabilistic information. It underlies many probabilistic logic programming languages such as Probabilistic Horn Abduction (Poole, 1993), PRISM (Sato, 1995; Sato and Kameya, 2001), Independent Choice Logic (Poole, 1997), Logic Programs with Annotated Disjunctions (Vennekens et al., 2004), ProbLog (De Raedt et al., 2007) and CP-logic (Vennekens et al., 2009).

A program in one of these languages defines a probability distribution over normal logic programs called worlds. This distribution is extended to queries and the probability of a query is obtained by marginalizing the joint distribution of the query and the programs. The languages following the distribution semantics

Email addresses: fabrizio.riguzzi@unife.it (Fabrizio Riguzzi), elena.bellodi@unife.it (Elena Bellodi), evelina.lamma@unife.it (Evelina Lamma), riccardo.zese@unife.it (Riccardo Zese) 
differ in the way they define the distribution over logic programs but have the same expressive power: there are transformations with linear complexity that can convert each one into the others (Vennekens and Verbaeten, 2003; De Raedt et al., 2008).

The distribution semantics was applied successfully in many domains (De Raedt et al., 2007; Sato and Kameya, 2001; Bellodi and Riguzzi, 2012) and various inference and learning algorithms are available for it (Kimmig et al., 2011; Riguzzi, 2009; Bellodi and Riguzzi, 2013).

In (Bellodi et al., 2011; Riguzzi et al., 2012a,b) we applied this approach to DLs obtaining DISPONTE for "DIstribution Semantics for Probabilistic ONTologiEs" (Spanish for "get ready"). The idea is to annotate axioms of a theory with a probability and assume that each axiom is independent of the others. A DISPONTE knowledge base (KB for short) defines a probability distribution over regular KBs (worlds) and the probability of a query is obtained from the joint probability of the worlds and the query. The DISPONTE semantics differs from previous proposals because it minimally extends the language and provides a unified framework for representing different types of probabilistic knowledge, from assertional to terminological knowledge. DISPONTE can be applied to any DL, here we present it for a prototypical expressive DL, $\mathcal{S H O \mathcal { N }}(\mathbf{D})$, that is the basis of OWL DL.

We also present the algorithm BUNDLE for "Binary decision diagrams for Uncertain reasoNing on Description Logic thEories" (Riguzzi et al., 2013a), that performs inference over DISPONTE DLs. BUNDLE exploits an underlying reasoner such as Pellet (Sirin et al., 2007) that returns explanations for queries. BUNDLE uses the inference techniques developed for probabilistic logic programs under the distribution semantics, in particular Binary Decision Diagrams (BDDs), for computing the probability of queries from a covering set of explanations. BUNDLE first finds explanations for the query and then encodes them in a BDD from which the probability can be computed in time linear in the size of the diagram.

BUNDLE's worst case complexity is high since the explanations may grow exponentially in number while the computation of the probability through Binary Decision Diagrams has \#P-complexity in the number of explanations. Nevertheless, we applied BUNDLE to various real world datasets and we found that it is able to handle domains of significant size.

The present paper extends previous work (Bellodi et al., 2011; Riguzzi et al., 2012a,b, 2013a) in various ways. First, we describe the DISPONTE semantics in more details and we discuss how a lower bound on the probability of a query can be computed, with the quality of the bound monotonically increasing as more time for inference is allowed. Moreover, we performed an extensive experimental analysis of BUNDLE in order to investigate its performance in practice. Finally, the paper includes a discussion on computational complexity and an extensive comparison with related work.

The paper is organized as follows. Section 2 introduces Description Logics with particular reference to $\mathcal{S H O I N}(\mathbf{D})$ while Section 3 discusses DISPONTE. Section 4 illustrates how to compute the probability of queries to DISPONTE DLs and Section 5 describes the BUNDLE algorithm. Section 6 discusses the complexity of reasoning and Section 7 related work. Section 8 shows the results of experiments with BUNDLE and, finally, Section 9 concludes the paper.

\section{Description Logics}

Description Logics (DLs) are knowledge representation formalisms that possess nice computational properties such as decidability and/or low complexity, see (Baader et al., 2003, 2008) for excellent introductions. DLs are particularly useful for representing ontologies and have been adopted as the basis of the Semantic Web.

While DLs are a fragment of predicate logic, they are usually represented using a syntax based on concepts and roles. A concept corresponds to a set of individuals of the domain while a role corresponds to a set of couples of individuals of the domain. In order to illustrate DLs, we describe $\mathcal{S H O \mathcal { N }} \mathcal{N}(\mathbf{D})$ as a prototype of expressive description logics. In the rest of the paper we use $\mathbf{A}, \mathbf{R}$ and $\mathbf{I}$ to indicate atomic concepts, atomic roles and individuals, respectively. A role is either an atomic role $R \in \mathbf{R}$ or the inverse $R^{-}$ of an atomic role $R \in \mathbf{R}$. We use $\mathbf{R}^{-}$to denote the set of all inverses of roles in $\mathbf{R}$. Concepts are defined as follows. Each $A \in \mathbf{A}, \perp$ and $\top$ are concepts and if $a \in \mathbf{I}$, then $\{a\}$ is a concept called a nominal. If $C, C_{1}$ 
and $C_{2}$ are concepts and $R \in \mathbf{R} \cup \mathbf{R}^{-}$, then $\left(C_{1} \sqcap C_{2}\right),\left(C_{1} \sqcup C_{2}\right)$ and $\neg C$ are concepts, as well as $\exists R$. $C$ and $\forall R . C$ and $\geq n R$ and $\leq n R$ for an integer $n \geq 0$.

An $R B$ ox $\mathcal{R}$ consists of a finite set of transitivity axioms $\operatorname{Trans}(R)$, where $R \in \mathbf{R}$, and role inclusion axioms $R \sqsubseteq S$, where $R, S \in \mathbf{R} \cup \mathbf{R}^{-}$. A TBox $\mathcal{T}$ is a finite set of concept inclusion axioms $C \sqsubseteq D$, where $C$ and $D$ are concepts. We use $C \equiv D$ to abbreviate $C \sqsubseteq D$ and $D \sqsubseteq C$. An $A B o x \mathcal{A}$ is a finite set of concept membership axioms $a: C$, role membership axioms $(a, b): R$, equality axioms $a=b$ and inequality axioms $a \neq b$, where $C$ is a concept, $R \in \mathbf{R}$ and $a, b \in \mathbf{I}$. A knowledge base $\mathcal{K}=(\mathcal{T}, \mathcal{R}, \mathcal{A})$ consists of a TBox $\mathcal{T}$, an $\operatorname{RBox} \mathcal{R}$ and an $\mathrm{ABox} \mathcal{A}$.

A $\mathcal{S H O I N}(\mathbf{D}) \mathrm{KB} \mathcal{K}$ is assigned a semantics in terms of interpretations $\mathcal{I}=\left(\Delta^{\mathcal{I}}, .^{\mathcal{I}}\right)$ where $\Delta^{\mathcal{I}}$ is a non-empty domain and ${ }^{\mathcal{I}}$ is the interpretation function that assigns an element in $\Delta^{\mathcal{I}}$ to each $a \in \mathbf{I}$, a subset of $\Delta^{\mathcal{I}}$ to each $A \in \mathbf{A}$ and a subset of $\Delta^{\mathcal{I}} \times \Delta^{\mathcal{I}}$ to each $R \in \mathbf{R}$. The mapping ${ }^{\mathcal{I}}$ is extended to all concepts (where $R^{\mathcal{I}}(x)=\left\{y \mid(x, y) \in R^{\mathcal{I}}\right\}$ and $\# X$ denotes the cardinality of the set $X$ ) as:

$$
\begin{aligned}
\left(R^{-}\right)^{\mathcal{I}} & =\left\{(y, x) \mid(x, y) \in R^{\mathcal{I}}\right\} \\
\top^{\mathcal{I}} & =\Delta^{\mathcal{I}} \\
\perp^{\mathcal{I}} & =\emptyset \\
\{a\}^{\mathcal{I}} & =\left\{a^{\mathcal{I}}\right\} \\
\left(C_{1} \sqcap C_{2}\right)^{\mathcal{I}} & =C_{1}^{\mathcal{I}} \cap C_{2}^{\mathcal{I}} \\
\left(C_{1} \sqcup C_{2}\right)^{\mathcal{I}} & =C_{1}^{\mathcal{I}} \cup C_{2}^{\mathcal{I}} \\
(\neg C)^{\mathcal{I}} & =\Delta^{\mathcal{I}} \backslash C^{\mathcal{I}} \\
(\forall R . C)^{\mathcal{I}} & =\left\{x \in \Delta^{\mathcal{I}} \mid R^{\mathcal{I}}(x) \subseteq C^{\mathcal{I}}\right\} \\
(\exists R . C)^{\mathcal{I}} & =\left\{x \in \Delta^{\mathcal{I}} \mid R^{\mathcal{I}}(x) \cap C^{\mathcal{I}} \neq \emptyset\right\} \\
(\geq n R)^{\mathcal{I}} & =\left\{x \in \Delta^{\mathcal{I}} \mid \# R^{\mathcal{I}}(x) \geq n\right\} \\
(\leq n R)^{\mathcal{I}} & =\left\{x \in \Delta^{\mathcal{I}} \mid \# R^{\mathcal{I}}(x) \leq n\right\}
\end{aligned}
$$

$\mathcal{S H O I N}(\mathbf{D})$ adds to $\mathcal{S H O I N}$ datatype roles, i.e., roles that map an individual to an element of a datatype such as integers, floats, etc. Then new concept definitions involving datatype roles are added that mirror those involving roles introduced above. We also assume that we have predicates over the datatypes.

The satisfaction of an axiom $E$ in an interpretation $\mathcal{I}=\left(\Delta^{\mathcal{I}},{ }^{\mathcal{I}}\right)$, denoted by $\mathcal{I}=E$, is defined as follows: (1) $\mathcal{I}=\operatorname{Trans}(R)$ iff $R^{\mathcal{I}}$ is transitive, (2) $\mathcal{I}=R \sqsubseteq S$ iff $R^{\mathcal{I}} \subseteq S^{\mathcal{I}}$, (3) $\mathcal{I} \models C \sqsubseteq D$ iff $C^{\mathcal{I}} \subseteq D^{\mathcal{I}}$, (4) $\mathcal{I} \models a: C$ iff $a^{\mathcal{I}} \in C^{\mathcal{I}}$, (5) $\mathcal{I}=(a, b): R$ iff $\left(a^{\mathcal{I}}, b^{\mathcal{I}}\right) \in R^{\mathcal{I}}$, (6) $\mathcal{I} \equiv a=b$ iff $a^{\mathcal{I}}=\bar{b}^{\mathcal{I}}$, (7) $\mathcal{I} \models a \neq b$ iff $a^{\mathcal{I}} \neq b^{\mathcal{I}}$. $\mathcal{I}$ satisfies a set of axioms $\mathcal{E}$, denoted by $\mathcal{I}=\mathcal{E}$, iff $\mathcal{I}=E$ for all $E \in \mathcal{E}$. An interpretation $\mathcal{I}$ satisfies a knowledge base $\mathcal{K}=(\mathcal{T}, \mathcal{R}, \mathcal{A})$, denoted $\mathcal{I}=\mathcal{K}$, iff $\mathcal{I}$ satisfies $\mathcal{T}, \mathcal{R}$ and $\mathcal{A}$. In this case we say that $\mathcal{I}$ is a model of $\mathcal{K}$.

A knowledge base $\mathcal{K}$ is satisfiable iff it has a model. An axiom $E$ is entailed by $\mathcal{K}$, denoted $\mathcal{K} \models E$, iff every model of $\mathcal{K}$ satisfies also $E$. A concept $C$ is satisfiable relative to $\mathcal{K}$ iff $\mathcal{K}$ has a model $\mathcal{I}$ such that $C^{\mathcal{I}} \neq \emptyset$.

A DL is decidable if the problem of checking the satisfiability of a $\mathrm{KB}$ is decidable. In particular, $\mathcal{S H O I N}(\mathbf{D})$ is decidable iff there are no number restrictions on non-simple roles. A role is non-simple iff it is transitive or it has transitive subroles.

A query over a KB is usually an axiom for which we want to test the entailment from the KB. The entailment test may be reduced to checking the unsatisfiability of a concept in the KB, i.e., the emptiness of the concept. For example, the entailment of the axiom $C \sqsubseteq D$ may be tested by checking the unsatisfiability of the concept $C \sqcap \neg D$.

\section{The DISPONTE Semantics for DLs}

DISPONTE applies the distribution semantics (Sato, 1995) of probabilistic logic programming to DLs. A program following this semantics defines a probability distribution over normal logic programs called worlds. Then the distribution is extended to queries and the probability of a query is obtained by marginalizing the joint distribution of the query and the programs. 


\subsection{Syntax and Intuition}

In DISPONTE, a probabilistic knowledge base $\mathcal{K}$ is a set of certain axioms or probabilistic axioms. Certain axioms take the form of regular DL axioms. Probabilistic axioms take the form

$$
p:: E
$$

where $p$ is a real number in $[0,1]$ and $E$ is a DL axiom.

The idea of DISPONTE is to associate independent Boolean random variables to the probabilistic axioms. By assigning values to every random variable we obtain a world, the set of axioms whose random variables are assigned the value 1.

Every formula obtained from a certain axiom is included in a world $w$. For each probabilistic axiom, we decide whether to include it or not in $w$. A world therefore is a non probabilistic KB that can be assigned a semantics in the usual way. A query is entailed by a world if it is true in every model of the world.

The probability $p$ can be interpreted as an epistemic probability, i.e., as the degree of our belief in axiom $E$. For example, a probabilistic concept membership axiom

$$
p:: a: C
$$

means that we have degree of belief $p$ in $C(a)$. The statement that Tweety flies with probability 0.9 can be expressed as

$$
0.9:: \text { tweety : Flies }
$$

A probabilistic concept inclusion axiom of the form

$$
p:: C \sqsubseteq D
$$

represents the fact that we believe in the truth of $C \sqsubseteq D$ with probability $p$. For example, the axiom

$$
0.9 \text { :: Bird } \sqsubseteq \text { Flies }
$$

means that birds fly with a $90 \%$ probability.

\subsection{Semantics}

We follow the approach of (Poole, 2000) and first give some definitions. An atomic choice is a couple $\left(E_{i}, k\right)$ where $E_{i}$ is the $i$ th probabilistic axiom and $k \in\{0,1\} . k$ indicates whether $E_{i}$ is chosen to be included in a world $(k=1)$ or not $(k=0)$.

A composite choice $\kappa$ is a consistent set of atomic choices, i.e., $\left(E_{i}, k\right) \in \kappa,\left(E_{i}, m\right) \in \kappa$ implies $k=m$ (only one decision is taken for each formula). The probability of a composite choice $\kappa$ is $P(\kappa)=$ $\prod_{\left(E_{i}, 1\right) \in \kappa} p_{i} \prod_{\left(E_{i}, 0\right) \in \kappa}\left(1-p_{i}\right)$, where $p_{i}$ is the probability associated with axiom $E_{i}$. A selection $\sigma$ is a total composite choice, i.e., it contains an atomic choice $\left(E_{i}, k\right)$ for every probabilistic axiom of the theory. A selection $\sigma$ identifies a theory $w_{\sigma}$ called a world in this way: $w_{\sigma}=\mathcal{C} \cup\left\{E_{i} \mid\left(E_{i}, 1\right) \in \sigma\right\}$ where $\mathcal{C}$ is the set of certain axioms. Let us indicate with $\mathcal{S}_{\mathcal{K}}$ the set of all selections and with $\mathcal{W}_{\mathcal{K}}$ the set of all worlds. The probability of a world $w_{\sigma}$ is $P\left(w_{\sigma}\right)=P(\sigma)=\prod_{\left(E_{i}, 1\right) \in \sigma} p_{i} \prod_{\left(E_{i}, 0\right) \in \sigma}\left(1-p_{i}\right) . P\left(w_{\sigma}\right)$ is a probability distribution over worlds, i.e., $\sum_{w \in \mathcal{W}_{\mathcal{K}}} P(w)=1$.

We can now assign probabilities to queries. Given a world $w$, the probability of a query $Q$ is defined as $P(Q \mid w)=1$ if $w \models Q$ and 0 otherwise. The probability of a query can be defined by marginalizing the joint probability of the query and the worlds:

$$
\begin{aligned}
P(Q) & =\sum_{w \in \mathcal{W}_{\mathcal{K}}} P(Q, w) \\
& =\sum_{w \in \mathcal{W}_{\mathcal{K}}} P(Q \mid w) p(w) \\
& =\sum_{w \in \mathcal{W}_{\mathcal{K}}: w \models Q} P(w)
\end{aligned}
$$


where (4) and (5) follow for the sum and product rule of the theory of probability respectively and (6) holds because $P(Q \mid w)=1$ if $w \models Q$ and 0 otherwise.

Example 1. Consider the following KB, inspired by the people+pets ontology proposed in (Patel-Schneider et al., 2003):

$$
\begin{aligned}
& 0.5 \quad: \quad \exists \text { has Animal.Pet } \sqsubseteq \text { NatureLover } \\
&(\text { kevin, fluffy }): \text { hasAnimal } \\
&(\text { kevin, tom }): \text { has Animal } \\
& \text { fluffy }: \text { Cat } \\
& \text { tom }: \text { Cat } \\
& 0.6 \quad: \text { Cat } \sqsubseteq \text { Pet }
\end{aligned}
$$

The KB indicates that the individuals that own an animal which is a pet are nature lovers with a 50\% probability and that kevin has the animals fluffy and tom. Fluffy and tom are cats and cats are pets with probability $60 \%$.

The KB has four worlds and the query axiom $Q=$ kevin: NatureLover is true in one of them, the one corresponding to the following selection:

$$
\{((7), 1),((12), 1)\}
$$

where each pair contains the axiom number and the value of its the selector. The other possible worlds, where the query $Q$ is false, are the following:

$$
\begin{aligned}
& \{((7), 1),((12), 0)\} \\
& \{((7), 0),((12), 1)\} \\
& \{((7), 0),((12), 0)\}
\end{aligned}
$$

The probability of the query is $P(Q)=0.5 \cdot 0.6=0.3$.

Example 2. Let us consider a slightly different knowledge base:

$$
\begin{aligned}
& \exists \text { hasAnimal.Pet } \sqsubseteq \text { NatureLover } \\
&(\text { kevin, fluffy }): \text { hasAnimal } \\
&(\text { kevin, tom }): \text { hasAnimal } \\
& 0.4 \quad:: \text { fluffy }: \text { Cat } \\
& 0.3 \quad:: \text { tom }: \text { Cat } \\
& 0.6 \quad: \text { Cat } \sqsubseteq \text { Pet }
\end{aligned}
$$

Here individuals that own an animal which is a pet are surely nature lovers and kevin has the animals fluffy and tom. Moreover, we believe in the fact that fluffy and tom are cats and that cats are pets with a certain probability.

This KB has eight worlds and the query axiom $Q=$ kevin: NatureLover is true in three of them, those corresponding to the following selections:

$$
\begin{aligned}
& \{((13), 1),((14), 0),((15), 1)\} \\
& \{((13), 0),((14), 1),((15), 1)\} \\
& \{((13), 1),((14), 1),((15), 1)\}
\end{aligned}
$$

so the probability is

$$
P(Q)=0.4 \cdot 0.7 \cdot 0.6+0.6 \cdot 0.3 \cdot 0.6+0.4 \cdot 0.3 \cdot 0.6=0.348
$$


Note that if the regular DL KB obtained by stripping the probabilistic annotations is inconsistent, there will be worlds that are inconsistent too. These worlds will entail the query trivially, as does the regular KB. A DISPONTE KB with inconsistent worlds should not be used to derive consequences, just as a regular DL $\mathrm{KB}$ that is inconsistent should not.

However, apparently contradictory probabilistic information is allowed. For example, the KB

$$
\begin{array}{rll}
0.9 & :: & \text { Bird } \sqsubseteq \text { Flies } \\
0.1 & :: & \text { tweety }: \text { Flies } \\
& & \text { tweety }: \text { Bird }
\end{array}
$$

states that the probability of flying of a bird is 0.9 and the probability of flying of tweety, a particular bird, is 0.1 . The two probabilistic statements are considered as independent evidence for tweety flying and are combined giving the probability 0.91 for the query tweety : Flies. In fact, this KB has four worlds and tweety: Flies is true in three of them, giving $P(Q)=0.9 \cdot 0.1+0.9 \cdot 0.9+0.1 \cdot 0.1=0.91$.

Thus knowledge about instances of the domain may reinforce general knowledge and vice-versa.

Example 3. The knowledge base

$$
\begin{aligned}
& \text { kevin }: \forall \text { friend.Person } \\
&(\text { kevin }, \text { laura }): \text { friend } \\
&(\text { laura }, \text { diana }): \text { friend } \\
& 0.4:: \text { Trans }(\text { friend })
\end{aligned}
$$

means that all individuals in the friend relationship with kevin are persons, that kevin is a friend of laura, that laura is a friend of diana and that given three individuals $a, b$ and $c$, there is a $40 \%$ probability that if $a$ is a friend of $b$ and $b$ is a friend of $c$ then $a$ is a friend of $c$. In particular, we have a $40 \%$ probability that, if kevin is a friend of laura and laura is a friend of diana, then kevin is a friend of diana. Since the first two are certain facts, then kevin is a friend of diana with a $40 \%$ probability and diana is a person also with a $40 \%$ probability.

The final report of the W3C Uncertainty Reasoning for the World Wide Web Incubator Group (URW3$\mathrm{XG}, 2008)$ discusses the challenge of reasoning with uncertain information on the World Wide Web. Moreover, it also highglights several use cases for the representation of uncertainty: combining knowledge from multiple, untrusted sources; discovering and using services in the presence of uncertain information on the user requirements and the service descriptions; recommending items to users; extracting and annotating information from the web; automatically performing tasks for users such as making an appointment, and handling healthcare and life sciences information and knowledge.

By introducing probability in an expressive description logic, such as $\mathcal{S H O I N}(\mathbf{D})$ that is one of the basis of OWL, we are able to tackle these problems as shown in the following example.

Example 4. Consider a KB similar to the one of Example 2 but where we have a single cat, fluffy. Suppose the user has the knowledge

$$
\begin{aligned}
& \exists \text { has Animal.Pet } \sqsubseteq \text { NatureLover } \\
& \text { (kevin, fluffy) : has Animal } \\
& \text { Cat } \sqsubseteq \text { Pet }
\end{aligned}
$$

and there are two sources of information with different reliability that provide the information that fluffy is a cat. On one source the user has a degree of belief of 0.4 , i.e., he thinks it is correct with a $40 \%$ probability, while on the other source he has a degree of belief 0.3, i.e. he thinks it is correct with a 30\% probability. The user can reason on this knowledge by adding the following statements to his KB:

$$
\begin{array}{ccc}
0.4 & : \text { fluffy : Cat } \\
0.3 & :: & \text { fluffy : Cat }
\end{array}
$$


The two statements represent independent evidence on fluffy being a cat.

The query axiom $Q=$ kevin: NatureLover is true in 3 out of the 4 worlds, those corresponding to the selections:

$$
\begin{aligned}
& \{((16), 1),((17), 1)\} \\
& \{((16), 1),((17), 0)\} \\
& \{((16), 0),((17), 1)\}
\end{aligned}
$$

So $P(Q)=0.4 \cdot 0.3+0.4 \cdot 0.7+0.6 \cdot 0.3=0.58$. This is reasonable if the two sources can be considered as independent. In fact, the probability comes from the disjunction of two independent Boolean random variables with probabilities respectively 0.4 and 0.3:

$$
\begin{aligned}
P(Q) & =P\left(X_{1} \vee X_{2}\right) \\
& =P\left(X_{1}\right)+P\left(X_{2}\right)-P\left(X_{1} \wedge X_{2}\right) \\
& =P\left(X_{1}\right)+P\left(X_{2}\right)-P\left(X_{1}\right) P\left(X_{2}\right) \\
& =0.4+0.3-0.4 \cdot 0.3=0.58
\end{aligned}
$$

\section{Inference}

We propose an approach for performing inference over DISPONTE DLs in which we first find explanations for the given query and then compute the probability of the query from them. In order to discuss the approach, we first need to introduce some definitions.

A composite choice $\kappa$ identifies a set of worlds $\omega_{\kappa}=\left\{w_{\sigma} \mid \sigma \in \mathcal{S}_{\mathcal{K}}, \sigma \supseteq \kappa\right\}$, the set of worlds whose selection is a superset of $\kappa$, i.e., the set of worlds "compatible" with $\kappa$. We define the set of worlds identified by a set of composite choices $K$ as $\omega_{K}=\bigcup_{\kappa \in K} \omega_{\kappa}$.

A composite choice $\kappa$ is an explanation for a query $Q$ if $Q$ is entailed by every world of $\omega_{\kappa}$. A set of composite choices $K$ is covering with respect to $Q$ if every world $w_{\sigma} \in \mathcal{W}_{\mathcal{K}}$ in which $Q$ is entailed is such that $w_{\sigma} \in \omega_{K}$. Two composite choices $\kappa_{1}$ and $\kappa_{2}$ are incompatible if their union is inconsistent. For example, the composite choices $\kappa_{1}=\left\{\left(E_{i}, 1\right)\right\}$ and $\kappa_{2}=\left\{\left(E_{i}, 0\right)\right\}$ are incompatible where $E_{i}$ is a probabilistic axiom.

A set $K$ of composite choices is pairwise incompatible if for all $\kappa_{1} \in K, \kappa_{2} \in K, \kappa_{1} \neq \kappa_{2}$ implies $\kappa_{1}$ and $\kappa_{2}$ are incompatible. For example

$$
K=\left\{\kappa_{1}, \kappa_{2}\right\}
$$

with

$$
\kappa_{1}=\left\{\left(E_{i}, 1\right)\right\}
$$

and

$$
\kappa_{2}=\left\{\left(E_{i}, 0\right),\left(E_{l}, 1\right)\right\}
$$

is pairwise incompatible.

We define the probability of a pairwise incompatible set of composite choices $K$ as

$$
P(K)=\sum_{\kappa \in K} P(\kappa)
$$

Two sets of composite choices $K_{1}$ and $K_{2}$ are equivalent if $\omega_{K_{1}}=\omega_{K_{2}}$, i.e., if they identify the same set of worlds. For example, $K$ in (18) is equivalent to

$$
K^{\prime}=\left\{\kappa_{1}^{\prime}, \kappa_{2}^{\prime}\right\}
$$

with

$$
\kappa_{1}^{\prime}=\left\{\left(E_{i}, 1\right)\right\}
$$

and

$$
\kappa_{2}^{\prime}=\left\{\left(E_{l}, 1\right)\right\}
$$


If $E$ is an axiom and $\kappa$ is a composite choice such that $\kappa \cap\{(E, 0),(E, 1)\}=\emptyset$, the split of $\kappa$ on $E$ is the set of composite choices $S_{\kappa, E}=\{\kappa \cup\{(E, 0)\}, \kappa \cup\{(E, 1)\}\}$. It is easy to see that $\kappa$ and $S_{\kappa, E}$ identify the same set of possible worlds, i.e., that $\omega_{\kappa}=\omega_{S_{\kappa, E}}$. For example, the split of $\kappa_{2}^{\prime}$ in (22) on $E_{i}$ contains $\kappa_{2}$ in (19) and $\left\{\left(E_{i}, 1\right),\left(E_{l}, 1\right)\right\}$.

Poole (2000) proved the following result.

Theorem 1. Given a finite set $K$ of finite composite choices, there exists a finite set $K^{\prime}$ of pairwise incompatible finite composite choices such that $K$ and $K^{\prime}$ are equivalent.

Proof. Given a finite set of finite composite choices $K$, there are two possibilities to form a new set $K^{\prime}$ of composite choices so that $K$ and $K^{\prime}$ are equivalent:

1. removing dominated elements: if $\kappa_{1}, \kappa_{2} \in K$ and $\kappa_{1} \subset \kappa_{2}$, let $K^{\prime}=K \backslash\left\{\kappa_{2}\right\}$.

2. splitting elements: if $\kappa_{1}, \kappa_{2} \in K$ are compatible (and neither is a superset of the other), there is a $(E, k) \in \kappa_{1} \backslash \kappa_{2}$. We replace $\kappa_{2}$ by the split of $\kappa_{2}$ on $E$. Let $K^{\prime}=K \backslash\left\{\kappa_{2}\right\} \cup S_{\kappa_{2}, E}$.

In both cases $\omega_{K}=\omega_{K^{\prime}}$. If we repeat these two operations until neither is applicable we obtain a splitting algorithm (see Figure 1) that terminates because $K$ is a finite set of finite composite choices. The resulting set $K^{\prime}$ is pairwise incompatible and is equivalent to the original set. For example, the splitting algorithm applied to $K^{\prime}(21)$ can return $K(18)$.

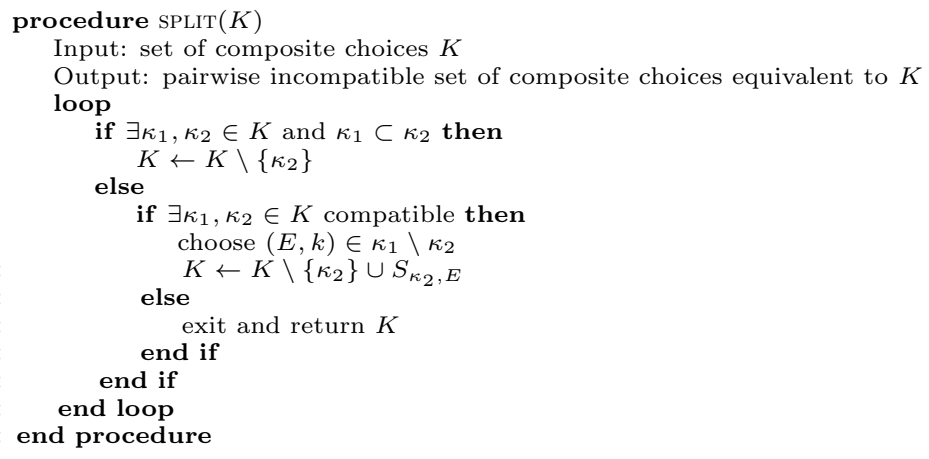

Figure 1: Splitting Algorithm.

Theorem 2 ((Poole, 1993)). If $K_{1}$ and $K_{2}$ are both pairwise incompatible finite sets of finite composite choices such that they are equivalent then $P\left(K_{1}\right)=P\left(K_{2}\right)$.

Proof. Consider the set $D$ of all axioms that appear in an atomic choice in either $K_{1}$ or $K_{2}$. This set is finite. Each composite choice in $K_{1}$ and $K_{2}$ has atomic choices for a subset of $D$. For both $K_{1}$ and $K_{2}$, we repeatedly replace each composite choice $\kappa$ of $K_{1}$ and $K_{2}$ with its split $S_{\kappa, E}$ on an $E$ from $D$ that does not appear in $\kappa$. This procedure does not change the total probability as the probabilities of $(E, 0)$ and $(E, 1)$ sum to 1.

At the end of this procedure the two sets of composite choices will be identical. In fact, any difference can be extended into a possible world belonging to $\omega_{K_{1}}$ but not to $\omega_{K_{2}}$ or vice versa, contradicting the hypothesis.

For example, $K$ in $(18)$ and $K^{\prime \prime}=\left\{\kappa_{1}^{\prime \prime}, \kappa_{2}^{\prime \prime}\right\}$ with $\kappa_{1}^{\prime \prime}=\left\{\left(E_{i}, 1\right),\left(E_{l}, 0\right)\right\}$ and $\kappa_{2}^{\prime \prime}=\left\{\left(E_{l}, 1\right)\right\}$ are equivalent and are both pairwise incompatible. Their probabilities are

$$
P(K)=p_{i}+\left(1-p_{i}\right) p_{l}=p_{i}+p_{l}-p_{i} p_{l}
$$

and

$$
P\left(K^{\prime \prime}\right)=p_{i}\left(1-p_{l}\right)+p_{l}=p_{i}+p_{l}-p_{i} p_{l}
$$


Note that if we compute the probability of $K^{\prime}$ in (21) with formula (20) we would obtain $p_{i}+p_{l}$ which is different from the probabilities of $K$ and $K^{\prime \prime}$ above, even if $K^{\prime}$ is equivalent to $K$ and $K^{\prime \prime}$, because $K^{\prime}$ is not pairwise incompatible.

We can thus define the probability $P(K)$ of a generic set of composite choices $K$ as $P(K)=P\left(K^{\prime}\right)$, where $K^{\prime}$ is a mutually incompatible set of composite choices that is equivalent to $K$, i.e., such that $\omega_{K^{\prime}}=\omega_{K}$. Given a query $Q$, the set $\left.K_{Q}=\left\{\sigma \mid \sigma \in \mathcal{S}_{\mathcal{K}} \wedge w_{\sigma}=Q\right\}\right)$ is a set of pairwise incompatible composite choices. Since $P(Q)=\sum_{\sigma \in K} P(\sigma)$, then $P(Q)=P\left(K_{Q}\right)$.

If $K^{\prime}$ is a set of explanations for $Q$ that is covering, then $K^{\prime}$ and $K_{Q}$ are equivalent so $P(Q)=P\left(K^{\prime}\right)$. Thus we do not have to generate all worlds where a query is true in order to compute its probability, finding a mutually incompatible covering set of explanations is enough.

Theorem 3. Given two finite sets of finite composite choices $K_{1}$ and $K_{2}$, if $K_{1} \subseteq K_{2}$, then $P\left(K_{1}\right) \leq P\left(K_{2}\right)$.

Proof. Let $K_{1}^{\prime}$ be the result of the application of the splitting algorithm to $K_{1}$. We can apply the same operations of the algorithm to $K_{2}$ obtaining $K=K_{1}^{\prime} \cup K^{\prime}$ for a certain $K^{\prime}$. At this point, we can continue applying the splitting algorithm. If there exists a $\kappa \in K$ and a $\kappa^{\prime} \in K$ such that $\kappa^{\prime} \subseteq \kappa$, at least one of $\kappa$ and $\kappa^{\prime}$ must not belong to $K_{1}^{\prime}$, otherwise $\kappa$ would have been removed when splitting $\bar{K}_{1}^{\prime}$. $\kappa$ is removed from $K$ while $\kappa^{\prime}$ remains. If there is a compatible couple $\kappa_{1}$ and $\kappa_{2}$ in $K$, we can assume that one of the two, say $\kappa_{2}$, does not belong to $K_{1}^{\prime}$, since otherwise it would have been split before. We add to $K$ the split of $\kappa_{2}$ on an atomic choice in $\kappa_{1}$ but not in $\kappa_{2}$.

Let $K_{2}^{\prime}$ be the results of the splitting algorithm so applied. $K_{2}^{\prime}$ is such that, for each element $\kappa_{1}$ of $K_{1}^{\prime}$, $K_{2}^{\prime}$ contains an element $\kappa_{2}$ such that $\kappa_{2} \subseteq \kappa_{1}$. Therefore, in the summation in (20), for each term in $P\left(K_{1}^{\prime}\right)$ there will be a term in $P\left(K_{2}^{\prime}\right)$ with a larger or equal value so $P\left(K_{1}^{\prime}\right) \leq P\left(K_{2}^{\prime}\right)$.

Thus, if $K$ is a finite set of finite explanations for a query $Q$ but we don't know if $K$ contains all possible explanations for $Q$, i.e., we don't know whether $K$ is covering, then $P(K)$ will be a lower bound of $P(Q)$. So we can compute progressively more accurate estimates from below of $P(Q)$ by considering an increasing number of explanations. Only when $K$ contains all possible explanations, then $P(K)=P(Q)$.

The problem of computing the probability of a query can thus be reduced to that of finding a covering set of explanations $K$ and then making it pairwise incompatible, so that the probability can be computed with the summation of (20). To obtain a pairwise incompatible set of explanations, the splitting algorithm can be applied.

Alternatively, given a covering set of explanations $K$ for a query $Q$, we can define the Disjunctive Normal Form (DNF) Boolean formula $f_{K}$ as

$$
f_{K}(\mathbf{X})=\bigvee_{\kappa \in K} \bigwedge_{\left(E_{i}, 1\right)} X_{i} \bigwedge_{\left(E_{i}, 0\right)} \overline{X_{i}}
$$

The variables $\mathbf{X}=\left\{X_{i} \mid\left(E_{i}, k\right) \in \kappa, \kappa \in K\right\}$ are independent Boolean random variables. The probability that $f_{K}(\mathbf{X})$ assumes value 1 is equal to the probability of $Q$.

We can now apply knowledge compilation to the propositional formula $f_{K}(\mathbf{X})$ (Darwiche and Marquis, 2002), i.e. translate it to a target language that allows answering queries in polynomial time. A target language that was found to give good performances is the one of Binary Decision Diagrams (BDD). From a BDD we can compute the probability of the query with a dynamic programming algorithm that is linear in the size of the BDD (De Raedt et al., 2007). Riguzzi (2009) showed that this approach is faster than the splitting algorithm.

A BDD for a function of Boolean variables is a rooted graph that has one level for each Boolean variable. A node $n$ in a BDD has two children: one corresponding to the 1 value of the variable associated with the level of $n$, indicated with $\operatorname{child}_{1}(n)$, and one corresponding the 0 value of the variable, indicated with $\operatorname{child}_{0}(n)$. When drawing BDDs, the 0-branch - the one going to $\operatorname{child}_{0}(n)$ - is distinguished from the 1-branch by drawing it with a dashed line. The leaves store either 0 or 1.

BDDs can be built by combining simpler BDDs using Boolean operators. While building BDDs, simplification operations can be applied that delete or merge nodes. Merging is performed when the diagram 


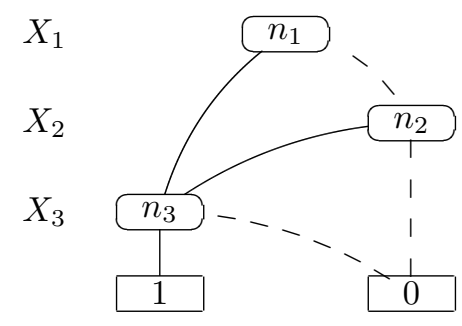

Figure 2: BDD for function (24).

contains two identical sub-diagrams, while deletion is performed when both arcs from a node point to the same node. In this way a reduced BDD is obtained, often with a much smaller number of nodes with respect to the original BDD. The size of the reduced BDD depends on the order of the variables: finding an optimal order is an NP-complete problem (Bollig and Wegener, 1996) and several heuristic techniques are used in practice by highly efficient software packages such as $\mathrm{CUDD}^{1}$. Alternative methods involve learning variable order from examples (Grumberg et al., 2003).

A BDD for the function

$$
f(\mathbf{X})=\left(X_{1} \wedge X_{3}\right) \vee\left(X_{2} \wedge X_{3}\right)
$$

is shown in Figure 2. A BDD performs a Shannon expansion of the Boolean formula $f_{K}(\mathbf{X})$, so that, if $X$ is the variable associated with the root level of a BDD, the formula $f_{K}(\mathbf{X})$ can be represented as $f_{K}(\mathbf{X})=X \wedge f_{K}^{X}(\mathbf{X}) \vee \bar{X} \wedge f_{K}^{\bar{X}}(\mathbf{X})$ where $f_{K}^{X}(\mathbf{X})\left(f_{K}^{\bar{X}}(\mathbf{X})\right)$ is the formula obtained by $f_{K}(\mathbf{X})$ by setting $X$ to $1(0)$. Now the two disjuncts are pairwise exclusive and the probability of $f_{K}(\mathbf{X})$ can be computed as $P\left(f_{K}(\mathbf{X})\right)=P(X) P\left(f_{K}^{X}(\mathbf{X})\right)+(1-P(X)) P\left(f_{K}^{\bar{X}}(\mathbf{X})\right)$. In other words, BDDs make the explanations pairwise incompatible. Figure 3 shows function PROB that implements the dynamic programming algorithm for computing the probability of a formula encoded as a BDD.

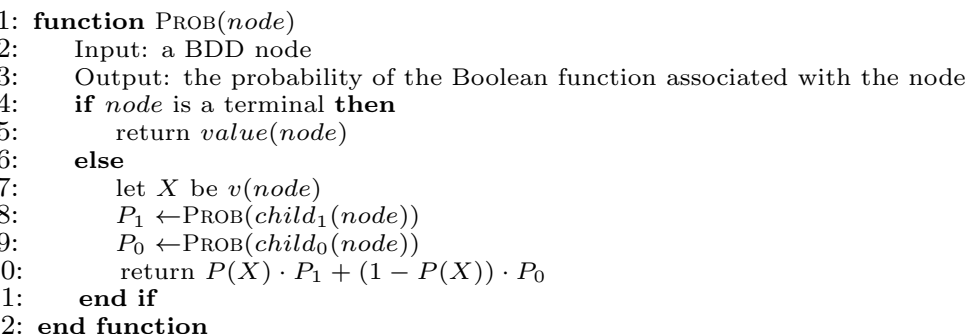

Figure 3: Function that computes the probability of a formula encoded as a BDD.

The function should also store the value of already visited nodes in a table so that, if a node is visited again, its probability can be retrieved from the table. For the sake of simplicity Figure 3 does not show this optimization but it is fundamental to achieve linear cost in the number of nodes, as without it the cost of the function PROB would be proportional to $2^{n}$ where $n$ is the number of Boolean variables.

Let us discuss inference on some examples.

Example 5. Let us consider the $K B$ of Example 2. A covering set of explanations for the query axiom $Q=$ kevin : NatureLover is $K=\left\{\kappa_{1}, \kappa_{2}\right\}$ where $\kappa_{1}=\{((13), 1),((15), 1)\}$ and $\kappa_{2}=\{((14), 1),((15), 1)\}$. If we associate the random variables $X_{1}$ to (13), $X_{2}$ to (14) and $X_{3}$ to (15), $f_{K}(\mathbf{X})$ is shown in (24) and the BDD associated with the set $K$ of explanations is shown in Figure 2.

\footnotetext{
${ }^{1}$ Available at http://vlsi.colorado.edu/ fabio/CUDD/
} 


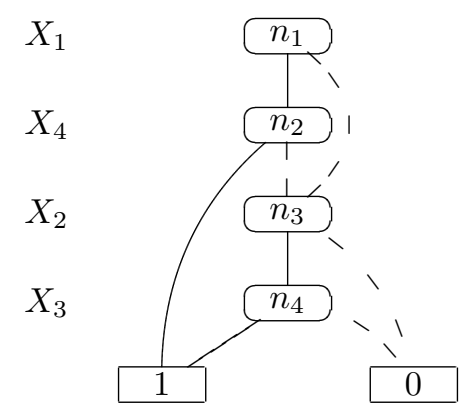

Figure 4: BDD for Example 6.

By applying the algorithm in Figure 3 we get

$$
\begin{aligned}
& \operatorname{Prob}\left(n_{3}\right)=0.6 \cdot 1+0.4 \cdot 0=0.6 \\
& \operatorname{Prob}\left(n_{2}\right)=0.4 \cdot 0.6+0.6 \cdot 0=0.24 \\
& \operatorname{Prob}\left(n_{1}\right)=0.3 \cdot 0.6+0.7 \cdot 0.24=0.348
\end{aligned}
$$

so $P(Q)=\operatorname{PROB}\left(n_{1}\right)=0.348$ which corresponds to the probability given by the semantics.

Example 6. Let us consider a slightly different knowledge base:

$$
\begin{aligned}
& \exists \text { has Animal.Pet } \sqsubseteq \text { NatureLover } \\
& \text { (kevin, fluffy) : has Animal } \\
& \text { (kevin,tom) : hasAnimal } \\
& 0.4 \text { :: fluffy : Dog } \\
& 0.3 \text { :: tom : Cat } \\
& 0.6 \text { :: Cat } \sqsubseteq \text { Pet } \\
& 0.5 \text { :: Dog } \sqsubseteq \text { Pet }
\end{aligned}
$$

A covering set of explanations for the query axiom $Q=$ kevin : NatureLover is $K=\left\{\kappa_{1}, \kappa_{2}\right\}$ where $\kappa_{1}=\{((25), 1),((28), 1)\}$ and $\kappa_{2}=\{((26), 1),((27), 1)\}$. If we associate the random variables $X_{1}$ to (25), $X_{2}$ to (26), $X_{3}$ to (27) and $X_{4}$ to (28), the BDD associated with the set $K$ of explanations is shown in Figure 4 .

By applying the algorithm in Figure 3 we get

$$
\begin{aligned}
& \operatorname{Prob}\left(n_{4}\right)=0.6 \cdot 1+0.4 \cdot 0=0.6 \\
& \operatorname{Prob}\left(n_{3}\right)=0.3 \cdot 0.6+0.7 \cdot 0=0.18 \\
& \operatorname{Prob}\left(n_{2}\right)=0.5 \cdot 1+0.5 \cdot 0.18=0.59 \\
& \operatorname{Prob}\left(n_{1}\right)=0.4 \cdot 0.59+0.6 \cdot 0.18=0.344
\end{aligned}
$$

so $P(Q)=\operatorname{PROB}\left(n_{1}\right)=0.344$.

\section{BUNDLE}

The BUNDLE algorithm computes the probability of queries from a probabilistic knowledge base that follows the DISPONTE semantics. It first finds a covering set of explanations for the query and then makes them pairwise exclusive by using BDDs. Finally, it computes the probability from the BDD by using function PROB in Figure 3. 
The problem of finding explanations for a query has been investigated by various authors (Kalyanpur, 2006; Kalyanpur et al., 2005b; Halaschek-Wiener et al., 2006; Kalyanpur et al., 2007). Schlobach and Cornet (2003) called it axiom pinpointing and considered it a non-standard reasoning service useful for tracing derivations and debugging ontologies. In particular, they define minimal axiom sets or MinAs for short.

Definition 1 (MinA). Let $\mathcal{K}$ be a $\mathrm{KB}$ and $Q$ an axiom that follows from it, i.e., $\mathcal{K}=Q$. We call a set $M \subseteq \mathcal{K}$ a minimal axiom set or $\operatorname{Min} A$ for $Q$ in $\mathcal{K}$ if $M=Q$ and $M$ is minimal w.r.t. set inclusion. The set of all possible MinAs is called $\operatorname{AlL}-\operatorname{Min} A s(Q, \mathcal{K})$. The problem of enumerating all MinAs is called MIN-A-ENUM.

The set of all MinAs can be used to derive a covering set of explanations. MIN-A-ENUM can be solved either with reasoner dependent (glass-box) approaches or reasoner independent (black-box) approaches (Kalyanpur et al., 2007). Glass-box approaches are built on existing tableau-based decision procedures and modify the internals of the reasoner. Black-box approaches use the DL reasoner solely as a subroutine and the internals of the reasoner do not need to be modified.

The techniques of (Kalyanpur et al., 2005a,b; Halaschek-Wiener et al., 2006; Kalyanpur et al., 2007) for axiom pinpointing have been integrated into the Pellet reasoner (Sirin et al., 2007). By default, Pellet solves MIN-A-ENUM with a hybrid glass/black-box approach: it finds a single MinA using a modified tableau algorithm and then finds all the other MinAs using a black box method (the hitting set tree algorithm). The method involves removing an axiom of the MinA from the KB and looking for alternative explanations. By repeating this process until the query is not entailed, the set of all explanations is found.

BUNDLE is based on Pellet and uses it for solving the MIN-A-ENUM problem. In the following, we first illustrate Pellet's algorithm for solving MIN-A-ENUM and then we show the whole BUNDLE algorithm.

\subsection{Axiom Pinpointing in Pellet}

Pellet exploits a tableau algorithm (Schmidt-Schauß and Smolka, 1991) that decides whether an axiom is entailed or not by a KB by refutation: axiom $E$ is entailed if $\neg E$ has no model in the KB. The algorithm works on completion graphs also called tableaux: they are ABoxes that can also be seen as graphs, where each node represents an individual $a$ and is labeled with the set of concepts $\mathcal{L}(a)$ it belongs to. Each edge $\langle a, b\rangle$ in the graph is labeled with the set of roles $\mathcal{L}(\langle a, b\rangle)$ to which the couple $(a, b)$ belongs. The algorithm starts from a tableau that contains the ABox of the KB and the negation of the axiom to be proved. For example, if the query is a membership one, $C(a)$, it adds $\neg C$ to the label of $a$. If we query for the emptyness (unsatisfiability) of a concept $C$, the algorithm adds a new anonymous node $a$ to the tableau and adds $C$ to the label of $a$. The axiom $C \sqsubseteq D$ can be proved by showing that $C \sqcap \neg D$ is unsatisfiable. The algorithm repeatedly applies a set of consistency preserving tableau expansion rules until a clash (i.e., a contradiction) is detected or a clash-free graph is found to which no more rules are applicable. In the following we describe the tableau algorithm used by Pellet. The algorithm is shown in Figure 5.

The rules used by Pellet to answer queries to $\mathcal{S H O I N}(\mathbf{D})$ knowledge bases are shown in Figure 8. Some of the rules are non-deterministic, i.e., they generate a finite set of tableaux. Thus the algorithm keeps a set of tableaux $T$. If a non-deterministic rule is applied to a graph $G$ in $T$, then $G$ is replaced by the resulting set of graphs. For example, if the disjunction $C \sqcup D$ is present in the label of a node, the rule $\rightarrow \sqcup$ generates two graphs, one in which $C$ is added to the node label and the other in which $D$ is added to the node label.

An event during the execution of the algorithm can be (Kalyanpur, 2006): 1) Add(C,a), the addition of a concept $C$ to $\mathcal{L}(a) ; 2) \operatorname{Add}(R,\langle a, b\rangle)$, the addition of a role $R$ to $\mathcal{L}(\langle a, b\rangle) ; 3) \operatorname{Merge}(a, b)$, the merging of the nodes $a, b ; 4) \neq(a, b)$, the addition of the inequality $a \neq b$ to the relation $\neq ; 5) \operatorname{Report}(g)$, the detection of a clash $g$. We use $\mathcal{E}$ to denote the set of events recorded during the execution of the algorithm. A clash is either:

- a couple $(C, a)$ where $C$ and $\neg C$ are present in the label of node $a$, i.e. $\{C, \neg C\} \subseteq \mathcal{L}(a)$;

- a couple $(\operatorname{Merge}(a, b), \neq(a, b))$, where the events $\operatorname{Merge}(a, b)$ and $\neq(a, b)$ belong to $\mathcal{E}$. 


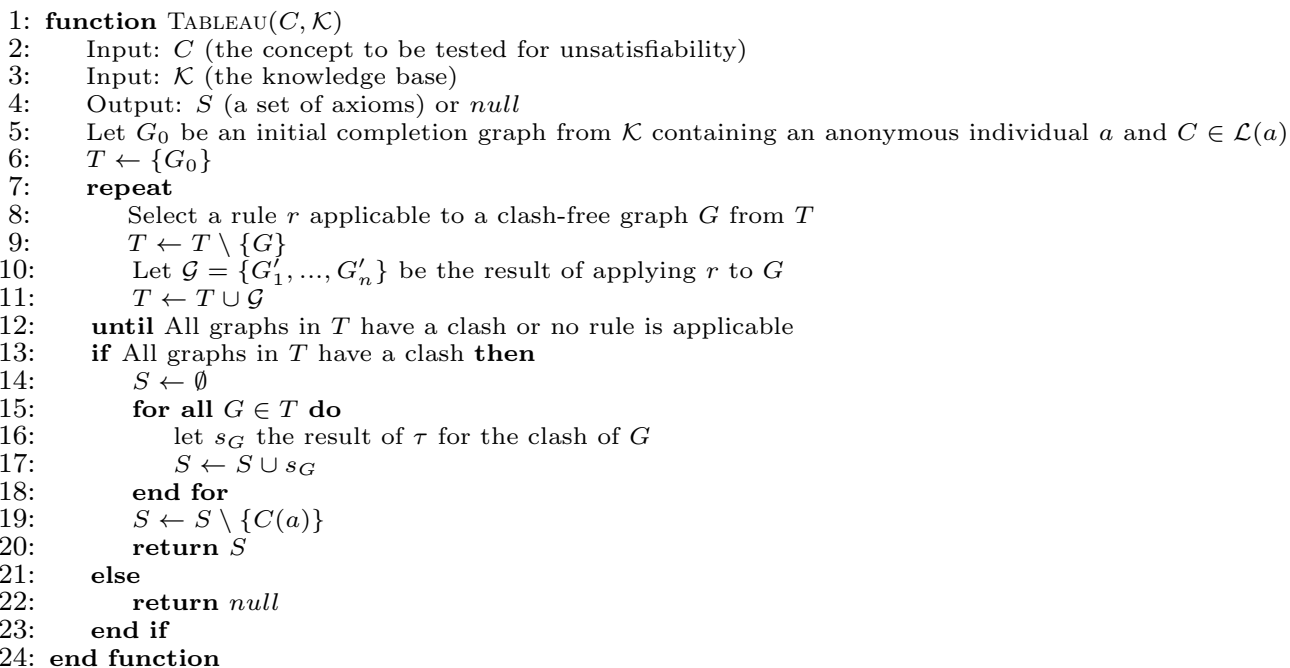

Figure 5: Tableau algorithm executed by Pellet.

Each time a clash is detected in a completion graph $G$, the algorithm stops applying rules to $G$. Once every completion graph in $T$ contains a clash or no more expansion rules can be applied to it, the algorithm terminates. If all the completion graphs in the final set $T$ contain a clash, the algorithm returns unsatisfiable as no model can be found. Otherwise, any one clash-free completion graph in $T$ represents a possible model for $C(a)$ and the algorithm returns satisfiable.

Each expansion rule updates as well a tracing function $\tau$, which associates sets of axioms with events in the derivation. For example, $\tau(A d d(C, a)),(\tau(A d d(R,\langle a, b\rangle)))$ is the set of axioms needed to explain the event $\operatorname{Add}(C, a)(A d d(R,\langle a, b\rangle))$. For the sake of brevity, we define $\tau$ for couples (concept, individual) and (role, couple of individuals) as $\tau(C, a)=\tau(A d d(C, a))$ and $\tau(R,\langle a, b\rangle)=\tau(A d d(R,\langle a, b\rangle))$ respectively. The function $\tau$ is initialized as the empty set for all the elements of its domain except for $\tau(C, a)$ and $\tau(R,\langle a, b\rangle)$ to which the values $\{a: C\}$ and $\{(a, b): R\}$ are assigned if $a: C$ and $(a, b): R$ are in the ABox respectively. The expansion rules (Figure 8) add axioms to values of $\tau$.

If $g_{1}, \ldots, g_{n}$ are the clashes, one for each tableau of the final set, the output of the algorithm TABLEAU is $S=\bigcup_{i \in\{1, \ldots, n\}} \tau\left(g_{i}\right) \backslash\{C(a)\}$ where $a$ is the anonymous individual initially assigned to $C$. However, this set may be redundant because additional axioms may also be included in $\tau$, e.g., during the $\rightarrow \leq$ rule, where axioms responsible for each of the $S$ successor edges are considered (Kalyanpur, 2006).

Thus $S$ is pruned using a black box approach shown in Figure 6 (Kalyanpur, 2006). This algorithm executes a loop on $S$ in which it removes an axiom from $S$ in each iteration and checks whether the concept $C$ turns satisfiable w.r.t. $S$, in which case the axiom is reinserted into $S$. The process continues until all axioms in $S$ have been tested and then returns $S$. Thus the algorithm for computing a single MinA, shown in Figure 7, first executes TABLEAU and then BLACKBoxPRUNing.

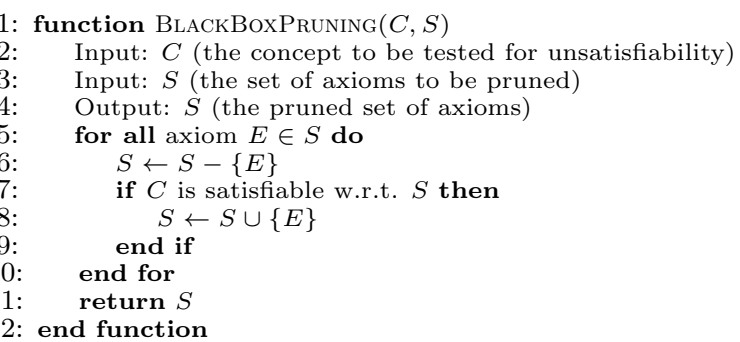

Figure 6: Black-Box pruning algorithm. 


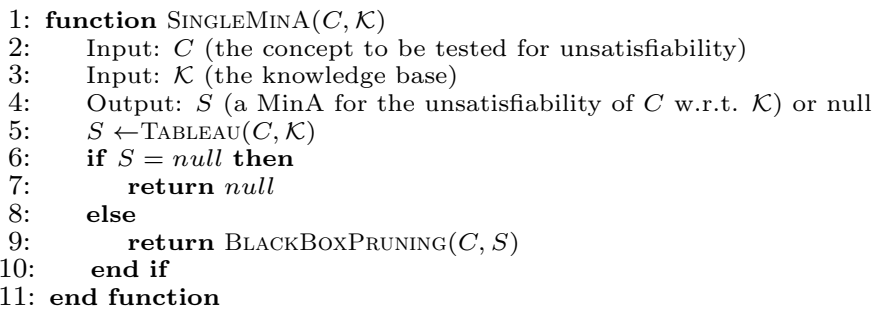

Figure 7: SingleMinA algorithm.

The output $S$ of SingleMinA is guaranteed to be a MinA, as established by the following theorem, where $\operatorname{All}-\operatorname{Min} A s(C, \mathcal{K})$ stands for the set of MinAs in which $C$ is unsatisfiable:

Theorem 4. (Kalyanpur, 2006) Let $C$ be an unsatisfiable concept w.r.t. $\mathcal{K}$ and let $S$ be the output of the algorithm SingleMinA with input $C$ and $\mathcal{K}$, then $S \in \operatorname{All}-\operatorname{MinAs}(C, \mathcal{K})$.

SingleMinA returns a single MinA. To solve Min-A-Enum, Pellet uses the hitting set algorithm (Reiter, 1987). The algorithm, described in detail in (Kalyanpur, 2006), starts from a MinA $S$ and initializes a labeled tree called Hitting Set Tree (HST) with $S$ as the label of its root $v$. Then it selects an arbitrary axiom $E$ in $S$, it removes it from $\mathcal{K}$, generating a new knowledge base $\mathcal{K}^{\prime}=\mathcal{K}-\{E\}$, and tests the unsatisfiability of $C$ w.r.t. $\mathcal{K}^{\prime}$. If $C$ is still unsatisfiable, we obtain a new explanation. The algorithm adds a new node $w$ in the tree and a new edge $\langle v, w\rangle$, then it assigns this new explanation to the label of $w$ and the axiom $E$ to the label of the edge. The algorithm repeats this process until the unsatisfiability test returns negative: in that case the algorithm labels the new node with $O K$, makes it a leaf, backtracks to a previous node, selects a different axiom to be removed from the KB and repeats these operations until the HST is fully built. The algorithm also eliminates extraneous unsatisfiability tests based on previous results: once a path leading to a node labeled $O K$ is found, any superset of that path is guaranteed to be a path leading to a node where $C$ is satisfiable, and thus no additional unsatisfiability test is needed for that path, as indicated by a $X$ in the node label. When the HST is fully built, all leaves of the tree are labeled with $O K$ or $X$. The distinct non leaf nodes of the tree collectively represent the set $\operatorname{ALL}-\operatorname{Min} \operatorname{As}(C, \mathcal{K})$.

The correctness and completeness of the hitting set algorithm are given by the following theorem.

Theorem 5. (Kalyanpur, 2006) Let $C$ be a concept unsatisfiable in $\mathcal{K}$ and let $\operatorname{ExpHST}(C, \mathcal{K})$ be the set of explanations returned by Pellet's hitting set algorithm. Then

$$
\operatorname{ExpHST}(C, \mathcal{K})=\operatorname{AlL}-\operatorname{Min} A s(C, \mathcal{K}) .
$$

Pellet's $\operatorname{ExpHST}(C, \mathcal{K})$ can also take as input a maximum number of explanations to be generated. If the limit is reached during the execution of the hitting set algorithm, Pellet stops and returns the set of explanations found so far.

\subsection{Overall BUNDLE}

The main BUNDLE function, shown in Figure 9, first builds a data structure PMap that associates each probabilistic DL axiom $E_{i}$ with its probability $p_{i}$. Then BUNDLE uses Pellet's $\operatorname{ExpHST}(C, \mathcal{K})$ function to compute the MinAs for the unsatisfiability of a concept $C$. BUNDLE exploits the version of this procedure in which we can specify the maximum number of explanations to be found.

Two data structures are initialized: $\operatorname{Var} A x$ is an array that maintains the association between Boolean random variables (whose index is the array index) and couples (axiom, probability), and $B D D$ stores a BDD. $B D D$ is initialized to the zero Boolean function.

Then BUNDLE performs two nested loops that build a BDD representing the set of explanations. To manipulate BDDs we used JavaBDD ${ }^{2}$ that is an interface to a number of underlying BDD manipulation packages. As the underlying package we used CUDD.

\footnotetext{
${ }^{2}$ Available at http://javabdd.sourceforge.net/
} 


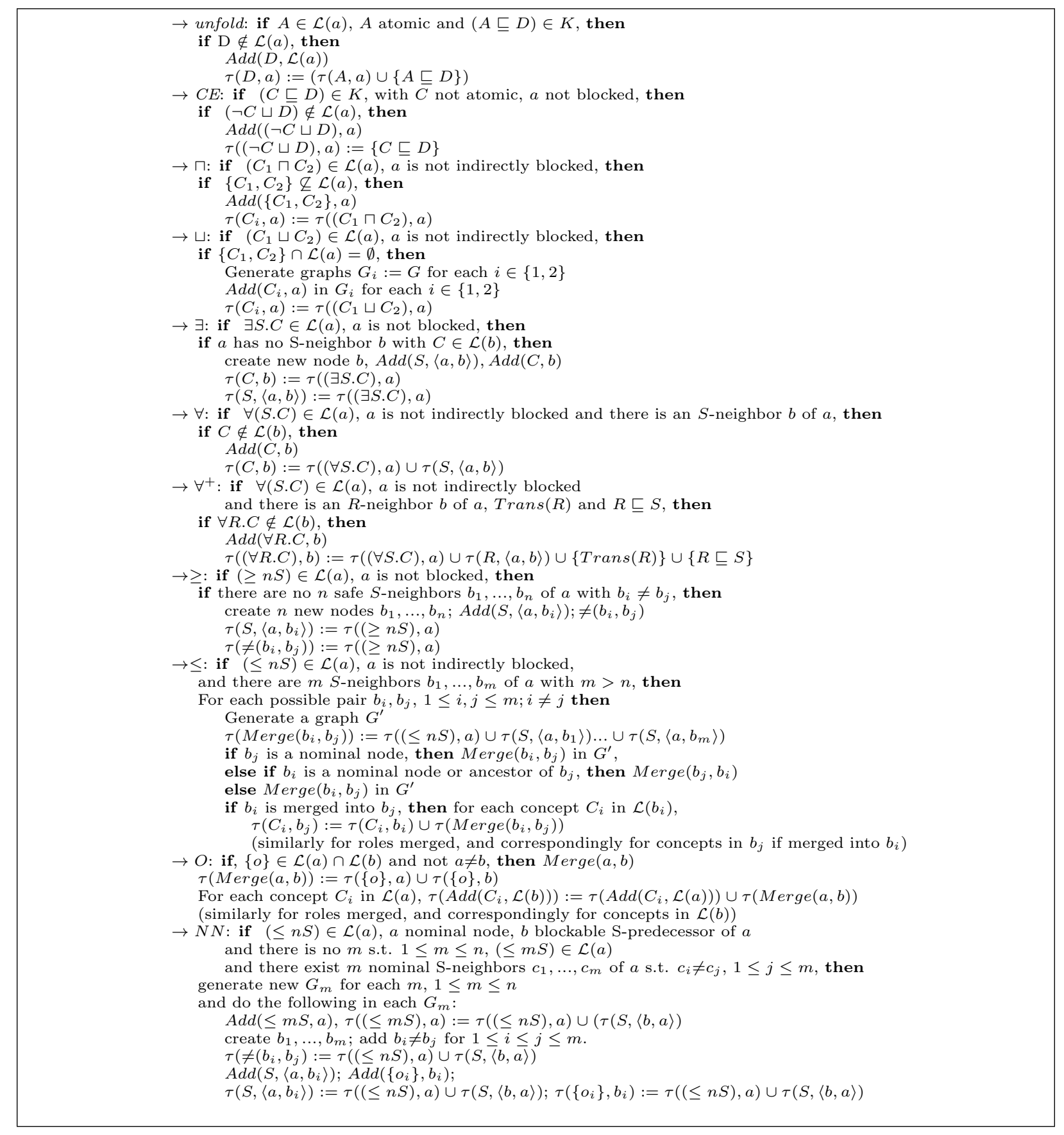

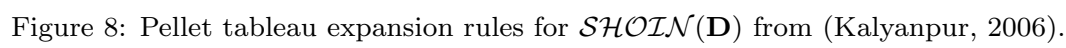




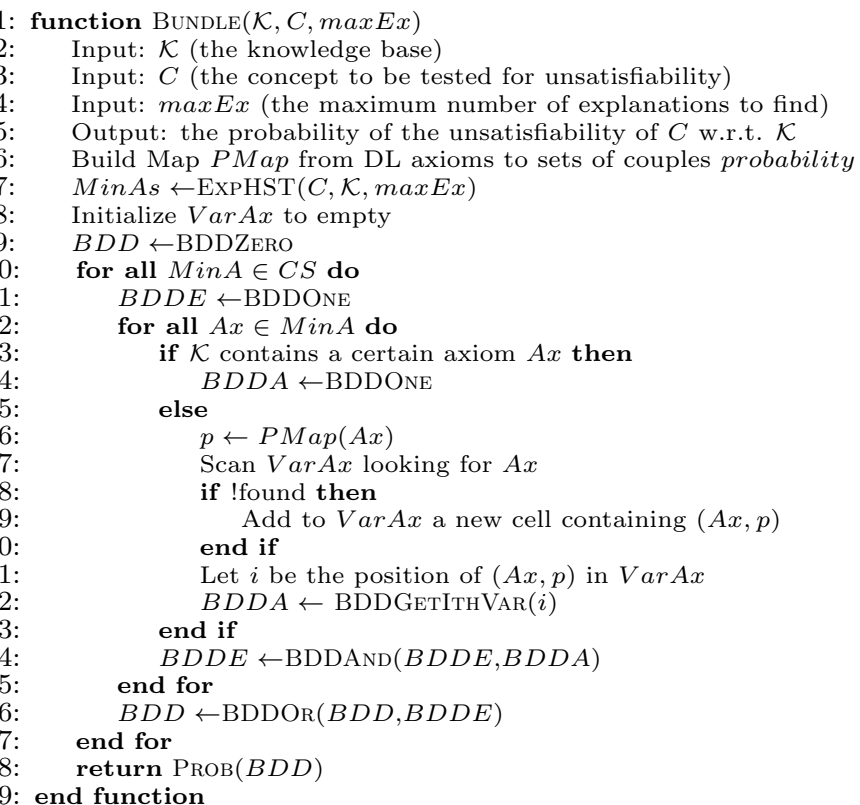

Figure 9: Function BundLE: computation of the probability of an axiom $Q$ given the KB $\mathcal{K}$.

In the outermost loop, BUNDLE combines BDDs for different explanations. In the inner loop, BUNDLE generates the BDD for a single explanation.

In the outermost loop, $B D D E$ is initialized to the one Boolean function. In the inner loop, the axioms of each MinA are considered one by one. If the axiom is certain, then the one Boolean function is conjoined with $B D D E$. Otherwise, the value $p$ associated with the axiom is extracted from PMap. The axiom is searched for in $\operatorname{Var} A x$ to see if it has already been assigned a random variable. If not, a cell is added to $\operatorname{Var} A x$ to store the couple. At this point we know the couple position $i$ in $\operatorname{Var} A x$ and so the index of its Boolean variable $X_{i}$. We obtain a BDD representing $X_{i}=1$ with BDDGeTIthVAR and we conjoin it with $B D D E$. After the two cycles, function PROB of Figure 3 is called over $B D D$ and its result is returned to the user.

We now prove BUNDLE correctness.

Theorem 6 (BUNDLE correctness). Given a DISPONTE KB $\mathcal{K}$, a query $Q$ and a limit maxEx for the number of explanations to find, the probability returned by BUNDLE, $\operatorname{BundLE}(\mathcal{K}, Q$, maxEx) is:

- a lower bound on $P(Q)$, i.e., $\operatorname{BUNDLE}(\mathcal{K}, Q, \max E x) \leq P(Q)$ if a maximum number of explanations is set

- equal to $P(Q)$, i.e., $\operatorname{Bundle}(\mathcal{K}, Q, \max E x)=P(Q)$ otherwise

Proof. Let $K$ be $\operatorname{ExpHST}(C, \mathcal{K}, \max E x)$. By Theorem 5

$$
K \subseteq \operatorname{AlL}-\operatorname{InstminAs}(C, \mathcal{K})
$$

if a maximum number of explanations is set and

$$
K=\operatorname{AlL}-\operatorname{InstaninAs}(C, \mathcal{K})
$$

otherwise. Since BUNDLE computes $P\left(f_{K}(\mathbf{X})\right)$ for the Boolean function

$$
f_{K}(\mathbf{X})=\bigvee_{\kappa \in K} \bigwedge_{(F, 1) \in \kappa} X_{F}
$$

the theorem holds. 


\section{Computational Complexity}

Jung and Lutz (2012) considered the problem of computing the probability of conjunctive queries to probabilistic databases in the presence of an ontology. Probabilities can occur only in the ABox while the TBox is certain. In the case where each ABox assertion is associated with a Boolean random variable independent of all the others, they prove that only very simple conjunctive queries can be answered in PTime, while most queries are \#P-hard when the ontology is a DL-Lite TBox and even when the ontology is a $\mathcal{E} \mathcal{L} \mathcal{I}$ TBox.

The class \#P (Valiant, 1979) describes counting problems associated with decision problems in NP. More formally, \#P is the class of function problems of the form "compute $f(x)$ ", where $f$ is the number of accepting paths of a nondeterministic Turing machine running in polynomial time. A prototypical \#P problem is the one of computing the number of satisfying assignments of a CNF Boolean formula. \#P problems were shown very hard. First, a \#P problem must be at least as hard as the corresponding NP problem. Second, Toda (1989) showed that a polynomial-time machine with a \#P oracle $(\mathrm{P} \# \mathrm{P})$ can solve all problems in $\mathrm{PH}$, the entire polynomial hierarchy.

The setting considered by Jung and Lutz (2012) is subsumed by DISPONTE as it is equivalent to having probabilistic axioms only in the ABox of a DISPONTE KB. So the complexity result provides a lower bound for DISPONTE.

In order to investigate the complexity of BUNDLE, we can consider the two problems that it solves for answering a query. The first one is axiom pinpointing. Its computational complexity has been studied in a number of works (Peñaloza and Sertkaya, 2009, 2010a,b). Baader et al. (2007) showed that there can be exponentially many MinAs for a very simple DL that allows only concept intersection.

Example 7. Given an integer $n \geq 1$, consider the TBox

$$
\mathcal{T}_{n}=\left\{B_{i-1} \sqsubseteq P_{i} \sqcap Q_{i}, P_{i} \sqsubseteq B_{i}, Q_{i} \sqsubseteq B_{i} \mid 1 \leq i \leq n\right\}
$$

The size of $\mathcal{T}_{n}$ is linear in $n$ and $\mathcal{T}_{n} \models B_{0} \sqsubseteq B_{n}$. There are $2^{n}$ MinAs for $B_{0} \sqsubseteq B_{n}$ since, for each $i, 1 \leq i \leq n$, it is enough to have $P_{i} \sqsubseteq B_{i}$ or $Q_{i} \sqsubseteq B_{i}$ in the set.

Thus the number of explanations for $\mathcal{S H O I N}(\mathbf{D})$ may be even larger. Given this fact, we do not consider complexity with respect to the input only. We say an algorithm runs in output polynomial time (Johnson et al., 1988) if it computes all the output in time polynomial in the overall size of the input and the output. Corollary 15 in (Peñaloza and Sertkaya, 2010b) shows that MIN-A-ENUM cannot be solved in output polynomial time for $D L$-Lite $_{\text {bool }}$ TBoxes unless $P=N P$. Since $D L$-Lite bool is a sublogic of $\mathcal{S H O I N}(\mathbf{D})$, this result also holds for $\mathcal{S H O \mathcal { N }}(\mathbf{D})$.

The second problem to be solved is computing the probability of a query, that can be seen as computing the probability of a sum-of-products, as explained below.

Definition 2 (sum-of-products). Given a Boolean expression $S$ in disjunctive normal form (DNF), or a sum-of-products, in the variables $\left\{v_{1}, \ldots, v_{n}\right\}$ and let $P\left(v_{i}\right)$ be the probability that $v_{i}$ is true with $i=1, \ldots, n$, compute the probability $P(S)$ of $S$, assuming all variables are independent.

This problem, called sum-OF-PRODUCTS, was shown to be \#P-hard (see e.g. Rauzy et al., 2003). Given that the input of the SUM-OF-PRODUCTS problem is of at least exponential size in the worst case, this means that computing the probability of an axiom from a $\mathcal{S H O I N}(\mathbf{D})$ knowledge base is intractable.

However, the algorithms that have been proposed for solving the two problems were shown to be able to work on inputs of real world size. For example, all MinAs have been found for various entailments over many real world ontologies within a few seconds (Kalyanpur, 2006; Kalyanpur et al., 2007). As regards the SUM-OF-PRODUCTS problem, algorithms based on BDDs were able to solve problems with hundred of thousand of variables (see e.g. the works on inference on probabilistic logic programs (De Raedt et al., 2007; Riguzzi, 2007, 2009; Riguzzi and Swift, 2010; Kimmig et al., 2011; Riguzzi and Swift, 2013)). Also methods for weighted model counting (Sang et al., 2005; Chavira and Darwiche, 2008) can be used to solve the SUM-OF-PRODUCTS problem.

Moreover, Section 8 below shows that in practice we can compute the probability of entailments on KBs of real-world size in BUNDLE, too. 


\section{Related Work}

Bacchus (1990) and Halpern (1990) discuss first-order logics of probability and distinguish statistical statements from statements about degrees of belief. Halpern (1990) presents two examples: the probability that a randomly chosen bird flies is 0.9 and the probability that Tweety (a particular bird) flies is 0.9 . The first statement captures statistical information about the world while the second captures a degree of belief. In order to express the second type of statement, called a "Type 2" statement, Halpern proposes the notation $w($ Flies $($ tweety $))=0.9$, where the function $w$ is used to indicate the probability, while in order to express the first, called a "Type 1" statement, he proposes the notation $w_{x}(\operatorname{Flies}(x) \wedge \operatorname{Bird}(x))=0.9 w_{x}(\operatorname{Bird}(x))$. The latter formula can be read as: given a randomly chosen $x$ in the domain, if $x$ is a bird, the probability that $x$ flies is 0.9 , or the conditional probability that $x$ flies given that it is a bird is 0.9. DISPONTE allows only Type 2 statements: $0.9::$ Bird $\sqsubseteq$ Flies means that birds fly with a probability of 0.9 .

Lutz and Schröder (2010) proposed Prob- $\mathcal{A L C}$ that is derived directly from Halpern's probabilistic first order logic and, as DISPONTE, considers only "Type 2" statements. They do so by adopting a possible world semantics and allowing concept expressions of the form $P_{\geq_{n}} C$ and $\exists P_{\geq_{n}} R$. $C$ in the language, the first expressing the set of individuals that belong to $C$ with probability greater than $n$ and the second the set of individuals $a$ connected to at least another individual $b$ of $C$ by role $R$ such that the probability of $R(a, b)$ is greater than $n$. Moreover, the ABox may contain expressions of the form $P_{\geq n} C(a)$ and $P_{\geq n} R(a, b)$ directly expressing degrees of belief, together with $P_{\geq n} \mathcal{A}$ where $\mathcal{A}$ is an ABox. Prob- $\mathcal{A L C}$ is 2-EXPTIME-hard even when probability values are restricted to 0 and 1 . Prob- $\mathcal{A L C}$ is complementary to DISPONTE $\mathcal{A L C}$ as it allows new concept and assertional expressions while DISPONTE allows probabilistic axioms.

Jung and Lutz (2012) presented an approach for querying probabilistic databases in the presence of an OWL2 QL ontology. Each assertion is assumed to be stored in a database and associated with probabilistic events. All atomic events are assumed to be probabilistically independent, resulting in a semantics very similar to the distribution semantics. The authors are interested in computing the answer probabilities to conjunctive queries. Probabilities can occur only in the data, but neither in the ontology nor in the query. Two types of ABoxes are considered: a general one where events are Boolean combinations of atomic events, and a restricted one, where each assertion is associated with a distinct atomic event. The latter setting is subsumed by DISPONTE. Only very simple conjunctive queries in the latter settings can be answered in PTime, while most queries are \#P-hard. The authors underline the general interest and usefulness of the approach for a wide range of applications including the management of data extracted from the web, machine translation, and dealing with data that arise from sensor networks.

Heinsohn (1994) proposed an extension of the description logic $\mathcal{A L C}$ that is able to express statistical information on the terminological knowledge such as partial concept overlapping. Similarly, Koller et al. (1997) presented a probabilistic description logic based on Bayesian networks that deals with statistical terminological knowledge. Both Heinsohn and Koller et al. do not allow probabilistic assertional knowledge about concept and role instances. Jaeger (1994) allows assertional knowledge about concept and role instances together with statistical terminological knowledge and combines the resulting probability distributions using cross-entropy minimization but does not allow Type 2 statements as DISPONTE.

Ding and Peng (2004) proposed a probabilistic extension of OWL that admits a translation into Bayesian networks. The semantics assigns a probability distribution $P(a)$ over individuals, i.e. $\sum_{a} P(a)=1$, and assigns a probability to a class $C$ as $P(C)=\sum_{a \in C} P(a)$, while we assign a probability distribution to worlds. PR-OWL (Costa et al., 2008; Carvalho et al., 2010) is an upper ontology that provides a framework for building probabilistic ontologies. It allows to use the first-order probabilistic logic MEBN (Laskey and Costa, 2005) for representing uncertainty in ontologies.

DISPONTE differs from Heinsohn (1994); Jaeger (1994); Koller et al. (1997); Ding and Peng (2004); Costa et al. (2008); Carvalho et al. (2010) because it minimally extends the language and provides a unified framework for representing different types of probabilistic knowledge: from assertional to terminological knowledge.

Luna et al. (2011) proposed an extension of $\mathcal{A L C}$, called $\mathrm{CR} \mathcal{A L C}$ that adopts an interpretation-based semantics. CR $\mathcal{A} \mathcal{L C}$ allows statistical axioms of the form $P(C \mid D)=\alpha$, which mean that for any element $x$ in the domain $\mathcal{D}$, the probability that $x$ is in $C$ given that it is in $D$ is $\alpha$, and of the form $P(R)=\beta$, 
which mean that for each couple of elements $x$ and $y$ in $\mathcal{D}$, the probability that $x$ is linked to $y$ by the role $R$ is $\beta$. The semantics of $\mathrm{CR} \mathcal{A} \mathcal{L C}$ is based on probability measures over the space of interpretations with a fixed domain. CR $\mathcal{A L C}$ allows to express Type 1 knowledge but not Type 2 . A CR $\mathcal{A} \mathcal{L C}$ KB $\mathcal{K}$ can be represented as a directed acyclic graph $G(\mathcal{K})$ in which a node represents a concept or a role and the edges represent the relations between them: if a concept $C$ directly uses concept $D$, then $D$ is a parent of $C$ in $G(\mathcal{K})$. Moreover, each restriction $\exists R . C$ and $\forall R . C$ is added as a node to $G(\mathcal{K}) . G(\mathcal{K})$ contains an edge from $R$ to each restriction directly using it and from each restriction to the concept $C$ appearing in it. $G(\mathcal{K})$ is a template for generating a ground graph given the domain $\mathcal{D}$ in which each node represents an instantiated logical atom $C(a)$ or $R(a . b)$. Inference can then be performed by a first order loopy belief propagation algorithm on the ground graph.

A different approach to the combination of DLs with probability is taken in (Giugno and Lukasiewicz, 2002; Lukasiewicz, 2002, 2008). The logic proposed in these papers is called P-SHIQ $(\mathbf{D})$ and allows both terminological probabilistic knowledge as well as assertional probabilistic knowledge about instances of concepts and roles. Terminological probabilistic knowledge is expressed using conditional constraints of the form $(D \mid C)[l, u]$ that informally mean "generally, if an object belongs to $C$, then it belongs to $D$ with a probability in $[l, u]$ ". PRONTO (Klinov, 2008; Klinov and Parsia, 2011) is a system that performs inference under this semantics. Similarly to (Jaeger, 1994), the terminological knowledge is interpreted statistically while the assertional knowledge is interpreted in an epistemic way by assigning degrees of beliefs to assertions. Moreover it also allows to express default knowledge about concepts that can be overridden in subconcepts and whose semantics is given by Lehmann's lexicographic default entailment (Lehmann, 1995). These works are based on Nilsson's probabilistic logic (Nilsson, 1986), where a probabilistic interpretation $\operatorname{Pr}$ defines a probability distribution over the set of interpretations Int. The probability of a logical formula $F$ according to $\operatorname{Pr}$, denoted $\operatorname{Pr}(F)$, is the sum of all $\operatorname{Pr}(I)$ such that $I \in I n t$ and $I \models F$. A probabilistic knowledge base $\mathcal{K}$ is a set of probabilistic formulas of the form $F \geq p$. A probabilistic interpretation $\operatorname{Pr}$ satisfies $F \geq p$ iff $\operatorname{Pr}(F) \geq p . \operatorname{Pr}$ satisfies $\mathcal{K}$, or $\operatorname{Pr}$ is a model of $\mathcal{K}$, iff $\operatorname{Pr}$ satisfies all $F \geq p \in \mathcal{K} . \operatorname{Pr}(F) \geq p$ is a tight logical consequence of $\mathcal{K}$ iff $p$ is the infimum of $\operatorname{Pr}(F)$ in the set of to all models $\operatorname{Pr}$ of $\mathcal{K}$. Computing tight logical consequences from probabilistic knowledge bases can be done by solving a linear optimization problem.

Nilsson's probabilistic logic differs from the distribution semantics: while the first considers sets of distributions, the latter computes a single distribution over possible worlds. Nilsson's logic allows weaker conclusions: considers a probabilistic ontology composed of the axioms $0.4:: a: C$ and $0.5:: b: C$ and a probabilistic theory composed of $C(a) \geq 0.4$ and $C(b) \geq 0.5$. The distribution semantics allows to say that $P(a: C \vee b: C)=0.7$, while with Nilsson's logic the lowest $p$ such that $\operatorname{Pr}(C(a) \vee C(b)) \geq p$ holds is 0.5. This is due to the fact that, while in Nilsson's logic no assumption about the independence of the statements is made, in the distribution semantics the probabilistic axioms are considered as independent, which allows to make stronger conclusions. However, this does not restrict expressiveness as one can specify any joint probability distribution over the logical ground atoms, possibly introducing new atoms if needed. This is testified by the fact that Bayesian networks can be encoded in probabilistic logic programs under the distribution semantics (Vennekens et al., 2004) and by the applications of the distribution semantics in a wide variety of domains (De Raedt et al., 2007; Sato and Kameya, 2001; Bellodi and Riguzzi, 2012). The use of Nilsson logic causes the approach of (Giugno and Lukasiewicz, 2002; Lukasiewicz, 2002, 2008) to reason with intervals of probability values, while with DISPONTE we work with point probabilities.

Other approaches, such as (d'Amato et al., 2008; Gottlob et al., 2011), combine a liteweight ontology language, DL-Lite and Datalog+/- respectively, with graphical models, Bayesian networks and Markov networks respectively. In both cases, an ontology is composed of a set of annotated axioms and a graphical model. The annotations are sets of assignments of random variables from the graphical model. The semantics is assigned by considering the possible worlds of the graphical model and by stating that an axiom holds in a possible world if the assignments in its annotation hold. The probability of a conclusion is then the sum of the probabilities of the possible worlds where the conclusion holds. Our approach provides a tighter integration of probability in ontologies as we do not rely on an additional graphical model. 


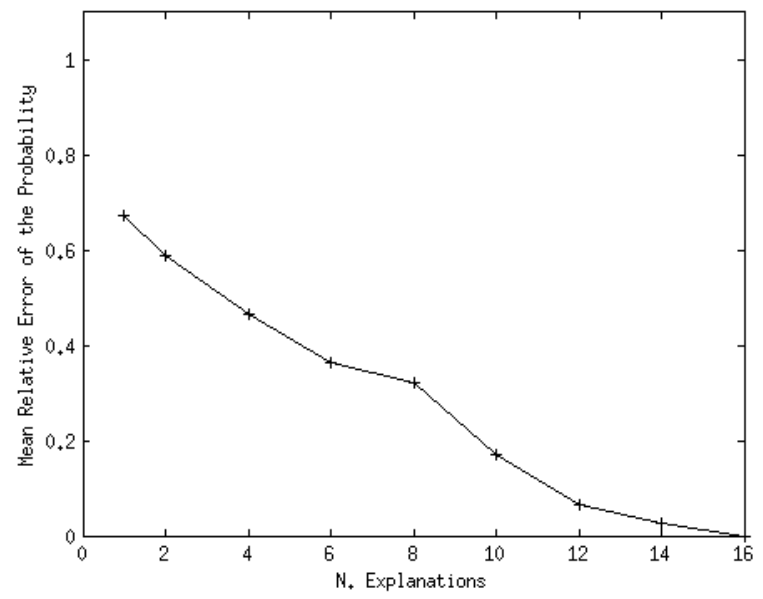

Figure 10: Mean relative error of the probability of queries as a function of the limit on the number of explanation varies.

\section{Experiments}

In order to test the performance of BUNDLE, we performed two experiments. All experiments have been performed on a Linux machine with a $3.10 \mathrm{GHz}$ Intel Xeon E5-2687W with 2GB (max) memory allotted to Java.

In the first one we consider how the execution time and the probability vary when imposing a limit on the number of explanations. We chose the $\mathrm{Grid}^{3} \mathrm{~KB}$ that is part of the myGrid project. The Grid KB has already been used for testing the performances of Pellet in (Kalyanpur et al., 2007). It describes the bioinformatics domain and contains concepts at a high level of abstraction. For the test, we used a version of the Grid KB with $\mathcal{S H O \mathcal { N }}$ expressiveness that contains 2838 axioms, 550 atomic concepts, 69 properties and 13 individuals, downloaded from the Tones repository ${ }^{4}$.

We associated a probability of 0.5 to each axiom of the KB and then we ran 100 different subclass queries. For generating the queries, we first built the hierarchy of the classes contained in the ontology. After that, for each query we randomly selected two classes that are connected in the hierarchy, so that each query had at least one explanation.

We first computed the correct probability of each query by using BUNDLE without a limit on the number of explanations in order to compute the correct probability. Then we ran each query several times, each time with an increased limit. The maximum number of explanations is 16: there are 20 queries with 16 explanations but most of the queries have a number of explanations between 1 and 5 . We computed the relative error $e$ between the correct probability $p$ of a query and the probability $p^{\prime}$ returned by BUNDLE with a limit on the number of explanations with the formula $e=\frac{p-p^{\prime}}{p}$. Then we averaged the relative error over all the queries.

In Figure 10 we show how the mean relative error varies with respect to the limit on the number of explanations, while Table 1 reports the execution times. The values are computed by averaging over all the 100 queries. The row of Table 1 with "_" in the first column contains the average execution time for BUNDLE without a limit on the number of explanations. Figure 11 shows the execution time as a function of the limit on the number of explanations. As can be seen, the quality of the answer increases as the limit on the number of explanations increases.

${ }^{3}$ http: //www.myGrid.org.uk/

${ }^{4}$ http://rpc295.cs.man.ac.uk: 8080/repository/browser 


\begin{tabular}{cc}
\hline $\begin{array}{c}\text { Limit on the } \\
\text { explanations }\end{array}$ & $\begin{array}{c}\text { Execution } \\
\text { time }(\mathrm{s})\end{array}$ \\
\hline 2 & 1.40 \\
\hline 4 & 1.44 \\
\hline 6 & 1.46 \\
\hline 8 & 1.49 \\
\hline 10 & 1.52 \\
\hline 12 & 1.55 \\
\hline 14 & 2.10 \\
\hline 16 & 9.36 \\
\hline- & 18.44 \\
\hline \hline
\end{tabular}

Table 1: Execution time depending on the limit on the number of explanations. The last row reports the time spent for finding the set of all explanations.

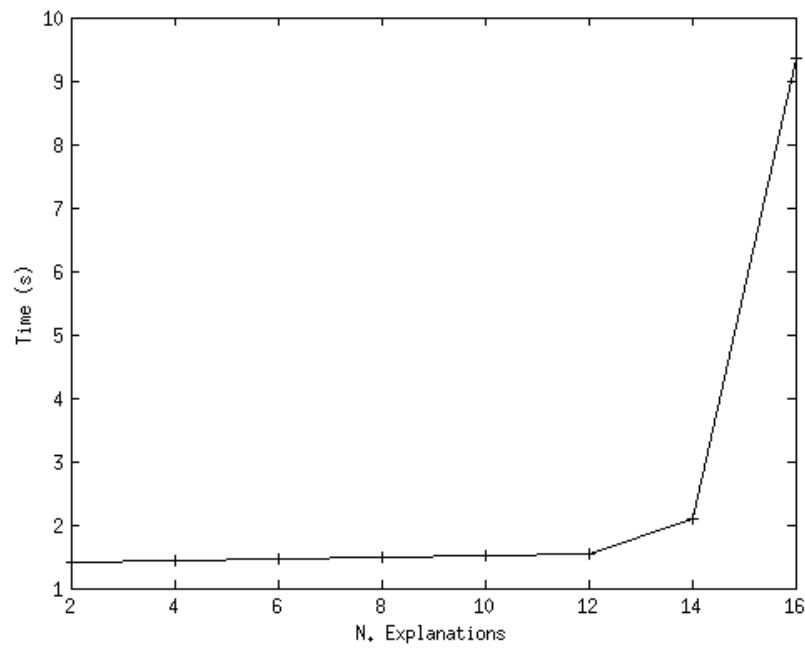

Figure 11: Execution time as the limit on the number of explanations to the queries varies. 


\begin{tabular}{|c|c|c|c|c|c|c|}
\hline \multirow[b]{2}{*}{ Dataset \& Infos } & & \multicolumn{5}{|c|}{ Dimension of Probabilistic TBox } \\
\hline & & 0 & 250 & 500 & 750 & 1000 \\
\hline Cell & time $(\mathrm{s})$ & 0.76 & 2.84 & 3.88 & 3.94 & 4.53 \\
\hline $\mathcal{A L E}+, 1263$ TBox's axioms & $\mathrm{TO}$ & 0 & 28 & 39 & 50 & 55 \\
\hline Teleost & time $(\mathrm{s})$ & 2.11 & 8.87 & 31.80 & 33.82 & 36.33 \\
\hline 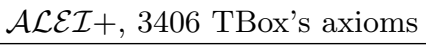 & $\mathrm{TO}$ & 0 & 7 & 32 & 32 & 44 \\
\hline NCI & time $(\mathrm{s})$ & 3.02 & 11.37 & 11.37 & 16.37 & 24.90 \\
\hline $\mathcal{A L E}+, 5423$ TBox's axioms & $\mathrm{TO}$ & 0 & 1 & 24 & 23 & 36 \\
\hline
\end{tabular}

Table 2: Average execution time for the queries to the Cell, Teleost and NCI KBs. The first column reports the expressiveness of each $\mathrm{KB}$ and the size of the non-probabilistic TBox.

In the second experiment we investigate the scalability of BUNDLE. Following the same method of evaluation presented by Klinov and Parsia (2011), we considered three different datasets: an extract from the Cell ${ }^{5}$ ontology, an extract from the NCI Thesaurus ${ }^{6}$ and an extract from the Teleost_anatomy ${ }^{7}$ ontology.

The Cell Ontology represents cell types of the prokaryotic, fungal, and eukaryotic organisms. The NCI ontology is an extract from the NCI Thesaurus that describes human anatomy. The Teleost_anatomy (Teleost for short) ontology is a multi-species anatomy ontology for teleost fishes.

For each of these KBs we considered the versions of increasing size used by Klinov and Parsia (2011): they added 250, 500, 750 and 1000 new probabilistic conditional constraints to the publicly available nonprobabilistic version of each ontology. We converted these KBs into DISPONTE by translating each conditional constraint $(D \mid C)[l, u]$ into the probabilistic axiom $u:: C \sqsubseteq D$ where $u$ is the upper limit of the conditional constraint interval. For each KB, we ran a set of 100 different random subclass queries without specifying a limit on the number of explanations. We created the queries as for the Grid KB: we built the hierarchy of each KB and we randomly selected two classes connected in the hierarchy for each query, so that each query had at least one explanation. We imposed a time limit of 5 minutes for BUNDLE to answer each query. If this limit is reached, BUNDLE's answer is "time-out".

In Table 2 we reported, for each version of the datasets, the average execution time and the number of queries that terminated with a time-out (TO). The averages are computed on the queries that did not end with a time-out. In addition, for each $\mathrm{KB}$ we reported its expressiveness and its number of non-probabilistic TBox axioms.

As can be seen, BUNDLE answers most queries in a few seconds. However, some queries have a very high complexity that causes BUNDLE to reach the time-out, confirming the complexity results. In these cases, since the time-out is reached during the computation of the explanations, limiting the number of explanations is necessary, obtaining a lower bound on the probability that becomes tighter as more explanations are allowed.

\section{Conclusions}

We have presented the DISPONTE semantics for probabilistic DLs that is inspired by the distribution semantics of probabilistic logic programming. We have discussed the application of DISPONTE to $\mathcal{S H O I N}(\mathbf{D})$, a prototype of an expressive DL. DISPONTE minimally extends the language to which it is applied and allows both assertional and terminological probabilistic knowledge. We have also presented the algorithm BUNDLE that is able to compute the probability of queries from DISPONTE KBs. BUNDLE has been tested on four real world KBs. The experiments show that BUNDLE is able to deal with ontologies of significant complexity, even if in some situations only an approximated answer takes a reasonable amount of time. BUNDLE is available for download from http://sites.unife.it/ml/bundle together with the datasets used in the experiments.

\footnotetext{
${ }^{5}$ http: //cellontology.org/

${ }^{6}$ http://ncit.nci.nih.gov/

${ }^{7}$ http://phenoscape.org/wiki/Teleost_Anatomy_Ontology
} 
In the future, we plan to consider extensions of the semantics including statistical or Type 1 knowledge in the terminology of Halpern (1990). Moreover, alternative approaches for inference will be considered, in particular reasoning algorithms returning a pinpointing formula such in (Baader and Peñaloza, 2010b,a): such a formula compactly encodes explanations for the query and can be converted directly into a BDD. We have also started to study the problem of learning the parameters (Riguzzi et al., 2013b) and we are planning to tackle the problem of learning the structure of probabilistic KB.

\section{References}

Baader, F., Calvanese, D., McGuinness, D. L., Nardi, D., Patel-Schneider, P. F. (Eds.), 2003. The Description Logic Handbook: Theory, Implementation, and Applications. Cambridge University Press.

Baader, F., Horrocks, I., Sattler, U., 2008. Description logics. In: Handbook of knowledge representation. Elsevier, Ch. 3, pp. 135-179.

Baader, F., Peñaloza, R., 2010a. Automata-based axiom pinpointing. J. Autom. Reasoning 45 (2), 91-129.

Baader, F., Peñaloza, R., 2010b. Axiom pinpointing in general tableaux. J. Log. Comput. 20 (1), 5-34.

Baader, F., Peñaloza, R., Suntisrivaraporn, B., 2007. Pinpointing in the description logic $\mathcal{E} \mathcal{L}^{+}$. In: Annual German Conference on AI. Vol. 4667 of LNCS. Springer, pp. 52-67.

Bacchus, F., 1990. Representing and reasoning with probabilistic knowledge - a logical approach to probabilities. MIT Press.

Bellodi, E., Lamma, E., Riguzzi, F., Albani, S., 2011. A distribution semantics for probabilistic ontologies. In: Bobillo, F., et al. (Eds.), International Workshop on Uncertain Reasoning for the Semantic Web. Vol. 778 of CEUR Workshop Proceedings. Sun SITE Central Europe.

Bellodi, E., Riguzzi, F., 2012. Experimentation of an expectation maximization algorithm for probabilistic logic programs. Intelligenza Artificiale 8 (1), 3-18.

Bellodi, E., Riguzzi, F., 2013. Expectation Maximization over binary decision diagrams for probabilistic logic programs. Intel. Data Anal. 17 (2), 343-363.

Bollig, B., Wegener, I., 1996. Improving the variable ordering of OBDDs is NP-complete. IEEE Trans. Computers 45 (9), 993-1002.

Carvalho, R. N., Laskey, K. B., Costa, P. C. G., 2010. PR-OWL 2.0 - bridging the gap to OWL semantics. In: Bobillo, F., et al. (Eds.), International Workshop on Uncertain Reasoning for the Semantic Web. Vol. 654 of CEUR Workshop Proceedings. Sun SITE Central Europe.

Chavira, M., Darwiche, A., 2008. On probabilistic inference by weighted model counting. Artif. Intell. 172 (6-7), 772-799.

Costa, P. C. G., Laskey, K. B., Laskey, K. J., 2008. PR-OWL: A Bayesian ontology language for the semantic web. In: International Workshop on Uncertainty Reasoning for the Semantic Web. Vol. 5327 of LNCS. Springer, pp. 88-107.

d'Amato, C., Fanizzi, N., Lukasiewicz, T., 2008. Tractable reasoning with Bayesian description logics. In: International Conference on Scalable Uncertainty Management. Vol. 5291 of LNCS. Springer, pp. 146-159.

Darwiche, A., Marquis, P., 2002. A knowledge compilation map. J. Artif. Intell. Res. 17, 229-264.

De Raedt, L., Demoen, B., Fierens, D., Gutmann, B., Janssens, G., Kimmig, A., Landwehr, N., Mantadelis, T., Meert, W., Rocha, R., Santos Costa, V., Thon, I., Vennekens, J., 2008. Towards digesting the alphabet-soup of statistical relational learning. In: NIPS*2008 Workshop on Probabilistic Programming.

De Raedt, L., Kimmig, A., Toivonen, H., 2007. ProbLog: A probabilistic Prolog and its application in link discovery. In: International Joint Conference on Artificial Intelligence. pp. 2462-2467.

Ding, Z., Peng, Y., 2004. A probabilistic extension to ontology language OWL. In: Hawaii International Conference On System Sciences. IEEE.

Giugno, R., Lukasiewicz, T., 2002. P-SHOQ(D): A probabilistic extension of SHOQ(D) for probabilistic ontologies in the semantic web. In: European Conference on Logics in Artificial Intelligence. Vol. 2424 of LNCS. Springer, pp. 86-97.

Gottlob, G., Lukasiewicz, T., Simari, G. I., 2011. Conjunctive query answering in probabilistic Datalog+/- ontologies. In: International Conference on Web Reasoning and Rule Systems. Vol. 6902 of LNCS. Springer, pp. 77-92.

Grumberg, O., Livne, S., Markovitch, S., 2003. Learning to order bdd variables in verification. J. Artif. Intell. Res. $18,83-116$.

Halaschek-Wiener, C., Kalyanpur, A., Parsia, B., 2006. Extending tableau tracing for ABox updates. Tech. rep., University of Maryland.

Halpern, J. Y., 1990. An analysis of first-order logics of probability. Artif. Intell. 46 (3), 311-350.

Heinsohn, J., 1994. Probabilistic description logics. In: Conference on Uncertainty in Artificial Intelligence. Morgan Kaufmann, pp. 311-318.

Jaeger, M., 1994. Probabilistic reasoning in terminological logics. In: International Conference on Principles of Knowledge Representation and Reasoning. pp. 305-316.

Johnson, D. S., Papadimitriou, C. H., Yannakakis, M., 1988. On generating all maximal independent sets. Inf. Process. Lett. $27(3), 119-123$

Jung, J. C., Lutz, C., 2012. Ontology-based access to probabilistic data with owl ql. In: Cudré-Mauroux, P., Heflin, J., Sirin, E., Tudorache, T., Euzenat, J., Hauswirth, M., Parreira, J. X., Hendler, J., Schreiber, G., Bernstein, A., Blomqvist, E. (Eds.), International Semantic Web Conference. Vol. 7649 of LNCS. Springer, pp. 182-197.

Kalyanpur, A., 2006. Debugging and repair of OWL ontologies. Ph.D. thesis, The Graduate School of the University of Maryland. 
Kalyanpur, A., Parsia, B., Cuenca-Grau, B., Sirin, E., 2005a. Tableaux tracing in SHOIN. Tech. Rep. 2005-66, University of Maryland.

Kalyanpur, A., Parsia, B., Horridge, M., Sirin, E., 2007. Finding all justifications of OWL DL entailments. In: Aberer, K., et al. (Eds.), International Semantic Web Conference. Vol. 4825 of LNCS. Springer, pp. 267-280.

Kalyanpur, A., Parsia, B., Sirin, E., Hendler, J. A., 2005b. Debugging unsatisfiable classes in OWL ontologies. J. Web Sem. $3(4), 268-293$.

Kimmig, A., Demoen, B., De Raedt, L., Costa, V. S., Rocha, R., 2011. On the implementation of the probabilistic logic programming language ProbLog. Theor. Prac. Log. Prog. 11 (2-3), 235-262.

Klinov, P., 2008. Pronto: A non-monotonic probabilistic description logic reasoner. In: European Semantic Web Conference. Vol. 5021 of LNCS. Springer, pp. 822-826.

Klinov, P., Parsia, B., 2011. A hybrid method for probabilistic satisfiability. In: Bjørner, N., Sofronie-Stokkermans, V. (Eds.), International Conference on Automated Deduction. Vol. 6803 of LNCS. Springer, pp. 354-368.

Koller, D., Levy, A. Y., Pfeffer, A., 1997. P-CLASSIC: A tractable probablistic description logic. In: National Conference on Artificial Intelligence. pp. 390-397.

Laskey, K. B., Costa, P. C. G., 2005. Of starships and Klingons: Bayesian logic for the 23rd century. In: Conference in Uncertainty in Artificial Intelligence. AUAI Press, pp. 346-353.

Lehmann, D. J., 1995. Another perspective on default reasoning. Ann. Math. Artif. Intell. 15 (1), $61-82$.

Lukasiewicz, T., 2002. Probabilistic default reasoning with conditional constraints. Ann. Math. Artif. Int. 34 (1-3), 35-88.

Lukasiewicz, T., 2008. Expressive probabilistic description logics. Artif. Int. 172 (6-7), 852-883.

Luna, J. E. O., Revoredo, K., Cozman, F. G., 2011. Learning probabilistic description logics: A framework and algorithms. In: Mexican International Conference on Artificial Intelligence. Vol. 7094 of LNCS. Springer, pp. 28-39.

Lutz, C., Schröder, L., 2010. Probabilistic description logics for subjective uncertainty. In: Lin, F., Sattler, U., Truszczynski, M. (Eds.), International Conference on Principles of Knowledge Representation and Reasoning. AAAI Press.

Nilsson, N. J., 1986. Probabilistic logic. Artif. Intell. 28 (1), 71-87.

Patel-Schneider, P, F., Horrocks, I., Bechhofer, S., 2003. Tutorial on OWL.

Peñaloza, R., Sertkaya, B., 2009. Axiom pinpointing is hard. In: International Workshop on Description Logics. Vol. 477 of CEUR Workshop Proceedings. CEUR-WS.org.

Peñaloza, R., Sertkaya, B., 2010a. Complexity of axiom pinpointing in the dl-lite family. In: International Workshop on Description Logics. Vol. 573 of CEUR Workshop Proceedings. CEUR-WS.org.

Peñaloza, R., Sertkaya, B., 2010b. Complexity of axiom pinpointing in the DL-Lite family of description logics. In: European Conference on Artificial Intelligence. IOS Press, pp. 29-34.

Poole, D., 1993. Probabilistic horn abduction and Bayesian networks. Artif. Intell. 64 (1).

Poole, D., 1997. The Independent Choice Logic for modelling multiple agents under uncertainty. Artif. Intell. 94 (1-2), 7-56.

Poole, D., 2000. Abducing through negation as failure: stable models within the independent choice logic. J. Log. Program. 44 (1-3), 5-35.

Rauzy, A., Châtelet, E., Dutuit, Y., Bérenguer, C., January 2003. A practical comparison of methods to assess sum-of-products. Reliability Engineering and System Safety 79 (1), 33-42.

Reiter, R., 1987. A theory of diagnosis from first principles. Artif. Intell. 32 (1), 57-95.

Riguzzi, F., 2007. A top down interpreter for LPAD and CP-logic. In: Congress of the Italian Association for Artificial Intelligence. Vol. 4733 of LNAI. Springer, pp. 109-120.

Riguzzi, F., 2009. Extended semantics and inference for the Independent Choice Logic. Log. J. IGPL 17 (6), 589-629.

Riguzzi, F., Bellodi, E., Lamma, E., 2012a. Probabilistic Datalog+/- under the distribution semantics. In: Kazakov, Y., Lembo, D., Wolter, F. (Eds.), International Workshop on Description Logics. Vol. 846 of CEUR Workshop Proceedings. Sun SITE Central Europe.

Riguzzi, F., Bellodi, E., Lamma, E., Zese, R., 2012b. Epistemic and statistical probabilistic ontologies. In: Bobillo, F., et al. (Eds.), International Workshop on Uncertain Reasoning for the Semantic Web. Vol. 900 of CEUR Workshop Proceedings. Sun SITE Central Europe, pp. 3-14.

Riguzzi, F., Bellodi, E., Lamma, E., Zese, R., 2013a. BUNDLE: A reasoner for probabilistic ontologies. In: Faber, W., Lembo, D. (Eds.), International Conference on Web Reasoning and Rule Systems. Vol. 7994 of LNCS. Springer, pp. $183-197$.

Riguzzi, F., Bellodi, E., Lamma, E., Zese, R., 2013b. Parameter learning for probabilistic ontologies. In: Faber, W., Lembo, D. (Eds.), International Conference on Web Reasoning and Rule Systems. Vol. 7994 of LNCS. Springer, pp. 265-270.

Riguzzi, F., Swift, T., 2010. Tabling and Answer Subsumption for Reasoning on Logic Programs with Annotated Disjunctions. In: Technical Communications of the International Conference on Logic Programming. Vol. 7 of Leibniz International Proceedings in Informatics (LIPIcs). Schloss Dagstuhl-Leibniz-Zentrum fuer Informatik, pp. $162-171$.

Riguzzi, F., Swift, T., 2013. Well-definedness and efficient inference for probabilistic logic programming under the distribution semantics. Theory Pract. Log. Program. 13 (Special Issue 02 - 25th Annual GULP Conference), $279-302$.

Sang, T., Beame, P., Kautz, H. A., 2005. Performing bayesian inference by weighted model counting. In: Veloso, M. M., Kambhampati, S. (Eds.), National Conference on Artificial Intelligence. AAAI Press / The MIT Press, pp. 475-482.

Sato, T., 1995. A statistical learning method for logic programs with distribution semantics. In: International Conference on Logic Programming. MIT Press, pp. 715-729.

Sato, T., Kameya, Y., 2001. Parameter learning of logic programs for symbolic-statistical modeling. J. Artif. Intell. Res. 15, 391-454.

Schlobach, S., Cornet, R., 2003. Non-standard reasoning services for the debugging of description logic terminologies. In: Gottlob, G., Walsh, T. (Eds.), International Joint Conference on Artificial Intelligence. Morgan Kaufmann, pp. 355-362.

Schmidt-Schauß, M., Smolka, G., 1991. Attributive concept descriptions with complements. Artif. Intell. 48 (1), 1-26. 
Sirin, E., Parsia, B., Cuenca-Grau, B., Kalyanpur, A., Katz, Y., 2007. Pellet: A practical OWL-DL reasoner. J. Web Sem. $5(2), 51-53$.

Toda, S., 1989. On the computational power of PP and +P. In: Annual Symposium on Foundations of Computer Science. IEEE Computer Society, pp. 514-519.

URW3-XG, 2008. Uncertainty reasoning for the World Wide Web, final report.

Valiant, L. G., 1979. The complexity of enumeration and reliability problems. SIAM J. Comp. 8 (3), 410-421.

Vennekens, J., Denecker, M., Bruynooghe, M., 2009. CP-logic: A language of causal probabilistic events and its relation to logic programming. Theory Pract. Log. Program. 9 (3), 245-308.

Vennekens, J., Verbaeten, S., 2003. Logic programs with annotated disjunctions. Tech. Rep. CW386, KU Leuven.

Vennekens, J., Verbaeten, S., Bruynooghe, M., 2004. Logic programs with annotated disjunctions. In: International Conference on Logic Programming. Vol. 3131 of LNCS. Springer, pp. 195-209. 